\title{
Frequency-Domain Techniques for Cerebral and Functional Near-Infrared Spectroscopy
}

\author{
Sergio Fantini* and Angelo Sassaroli \\ Department of Biomedical Engineering, Tufts University, Medford, MA, United States
}

This article reviews the basic principles of frequency-domain near-infrared spectroscopy (FD-NIRS), which relies on intensity-modulated light sources and phase-sensitive optical detection, and its non-invasive applications to the brain. The simpler instrumentation and more straightforward data analysis of continuous-wave NIRS (CW-NIRS) accounts for the fact that almost all the current commercial instruments for cerebral NIRS have embraced the CW technique. However, FD-NIRS provides data with richer information content, which complements or exceeds the capabilities of CW-NIRS. One example is the ability of FD-NIRS to measure the absolute optical properties (absorption and

OPEN ACCESS

Edited by:

Ning Liu,

Stanford University, United States

Reviewed by:

Robert James Cooper University College London,

United Kingdom

Felix Scholkmann,

University Hospital Zürich, Switzerland

Thomas D. O'Sullivan, University of Notre Dame,

United States

*Correspondence:

Sergio Fantini

sergio.fantini@tufts.edu

Specialty section:

This article was submitted to

Brain Imaging Methods,

a section of the journal

Frontiers in Neuroscience

Received: 10 December 2019

Accepted: 16 March 2020

Published: 07 April 2020

Citation:

Fantini S and Sassaroli A (2020)

Frequency-Domain Techniques for Cerebral and Functional

Near-Infrared Spectroscopy.

Front. Neurosci. 14:300.

doi: 10.3389/fnins.2020.00300 reduced scattering coefficients) of tissue, and thus the absolute concentrations of oxyhemoglobin and deoxyhemoglobin in brain tissue. This article reviews the measured values of such optical properties and hemoglobin concentrations reported in the literature for animal models and for the human brain in newborns, infants, children, and adults. We also review the application of FD-NIRS to functional brain studies that focused on slower hemodynamic responses to brain activity (time scale of seconds) and faster optical signals that have been linked to neuronal activation (time scale of $100 \mathrm{~ms}$ ). Another example of the power of FD-NIRS data is related to the different regions of sensitivity featured by intensity and phase data. We report recent developments that take advantage of this feature to maximize the sensitivity of non-invasive optical signals to brain tissue relative to more superficial extracerebral tissue (scalp, skull, etc.). We contend that this latter capability is a highly appealing quality of FD-NIRS, which complements absolute optical measurements and may result in significant advances in the field of non-invasive optical sensing of the brain.

\footnotetext{
Keywords: near-infrared spectroscopy, diffuse optical imaging, frequency domain, cerebral hemodynamics, brain activation, fast optical signal, depth sensitivity
}

\section{INTRODUCTION}

Diffuse optical imaging, in the near-infrared spectral band (wavelength range: 600-1000 nm), affords non-invasive sensing of the human brain through the intact scalp and skull. The associated instrumentation can be sufficiently lightweight and compact to be applicable at the bedside, in an ambulance, on the field (accident scene, sports venue, battlefield, etc.), in every-day settings, and even worn by subjects while they engage in normal activities. These enormous practical advantages of non-invasive diffuse optical imaging, combined with a strong sensitivity to cerebral hemodynamics and oxygenation, compensate intrinsic limitations in penetration depth and spatial resolution, and account for the large number of applications and the growing interest 
in this technology. Several review articles cover a variety of topics in cerebral near-infrared spectroscopy (NIRS), including a historical overview (Ferrari and Quaresima, 2012), description of instrumentation and methodology (Scholkmann et al., 2014b), clinical applications (Irani et al., 2007; Smith, 2011; Obrig, 2014; Yang et al., 2019), brain oximetry in newborns and adults (Wolf et al., 2012; Nielsen, 2014; la Cour et al., 2018), and critical perspectives (Fantini et al., 2018; Quaresima and Ferrari, 2019).

NIRS can be performed using methods in continuous wave (CW) (constant illumination), time domain (TD) (pulsed illumination and time-resolved detection), or frequency domain (FD) (intensity-modulated illumination and phase-resolved detection). Review articles have been devoted to functional NIRS (fNIRS) based on CW methods (Scholkmann et al., 2014b), the most common technique and the one embraced by almost all commercial instruments, and TD methods (Torricelli et al., 2014). No review articles have been previously devoted specifically to cerebral and functional FD-NIRS, and this article intends to fill this gap. However, the general topic of FDNIRS and its applications to biological tissue are covered in book chapters (Fantini et al., 1997; Cerussi and Tromberg, 2003; Srinivasan et al., 2011; Fantini and Sassaroli, 2016), review and tutorial articles (Chance et al., 1998b; O'Sullivan et al., 2012), and extended sections in biomedical optics books (Tuchin, 2007; Wang and Wu, 2007; Bigio and Fantini, 2016, Chapter 12).

As mentioned, CW techniques are by far the most commonly used, especially in the commercial space; in fact, to the best of our knowledge, while more than ten companies manufacture CW-NIRS systems worldwide, at the time of writing only one company (Hamamatsu Photonics, Japan) manufactures TD-NIRS systems, and only one company (ISS, Inc., United States) manufactures FD-NIRS systems. In the research arena, however, TD-NIRS and FD-NIRS are employed by several groups worldwide.

One question that is investigated in this article is the following: what is the advantage of using FD techniques for cerebral and functional NIRS, given its added instrumental complexity compared to CW-NIRS? This question will be explored by first describing the information content of FD data and how it enriches and differs from that of CW data, and then by describing the way in which FD data have been used in the field of NIRS studies of the brain. Following this description and a review of the literature, we will express our views on the potential role of FD techniques in cerebral and functional NIRS as this field is growing and evolving.

\section{FREQUENCY-DOMAIN NEAR-INFRARED SPECTROSCOPY (FD-NIRS)}

\section{The FD-NIRS Data: DC Intensity, AC Amplitude, Phase}

The basic idea of FD-NIRS is to use intensity-modulated illumination and phase-sensitive detection. Sinusoidal modulation, or a sinusoidal component of the modulated signal, at a given angular frequency $\omega$ is typical considered, so that the time-dependence of the FD-NIRS signal is described by $e^{-i \omega t}$. As a result, the optical signal measured in FD-NIRS consists of an average, or direct current (DC), intensity, and by a phasor described by an amplitude, or alternating current (AC) intensity, and a phase $(\varphi)$ :

$$
F D-N I R S \text { signal }=D C+A C e^{i \varphi} e^{-i \omega t}
$$

Typical modulation frequencies employed in the application of FD-NIRS to biological tissue are of the order of $100 \mathrm{MHz}$. Such a choice is related to the photon mean time of flight $(t)$, which is of the order of nanoseconds for light propagation in typical biological tissues and for distances of centimeters between the illumination and detection points. Since the phase is related to the mean time of flight by the relationship $\varphi \approx \omega t$, a measurable phase of the order of 1 rad requires a modulation frequency $[f=$ $\omega /(2 \pi)]$ of the order of $100 \mathrm{MHz}$. Lower modulation frequencies (say, $10 \mathrm{MHz}$ or less) would cause a smaller phase shift that may be close to the limit of phase detectability. On the contrary, higher modulation frequencies (say, $1 \mathrm{GHz}$ or more) would result in smaller AC amplitudes, possibly below the noise level, because of an increasing attenuation of the FD phasor at higher frequencies.

The solution of the diffusion equation in the frequency domain shows that the energy density distribution in turbid media resulting from intensity-modulated illumination can be described in terms of photon-density waves (PDWs) (Fishkin and Gratton, 1993; Tromberg et al., 1993). For a point-source in an infinite medium, PDWs are overdamped spherical waves. For typical optical properties of biological tissue in the near-infrared (absorption coefficient: $\mu_{a}=0.1 \mathrm{~cm}^{-1}$; reduced scattering coefficient: $\mu_{s}^{\prime}=10 \mathrm{~cm}^{-1}$ ), and for a typical modulation frequency of $100 \mathrm{MHz}$, the wavelength of the photon density waves is about $25 \mathrm{~cm}$, while its attenuation length (i.e., the distance over which the energy density decreases by a factor of $e$ ) is about $0.6 \mathrm{~cm}$. Therefore, PDWs are observable only over a fraction of their wavelength, rendering them analogous to near field waves (Bigio and Fantini, 2016, Section 12.4).

For typical modulation frequencies used in FD-NIRS and for typical optical properties of tissue in the near-infrared, DC intensity and AC amplitude carry a similar information content. For example, they feature a similar decay at increasing distances from the light source (although the AC attenuation is stronger than the DC attenuation), and a similar dependence to localized or homogeneous changes in the optical properties of tissue. However, the AC amplitude offers the practical advantage of being relatively insensitive to room light, or to any nonmodulated stray light, so that it is often used instead of DC intensity when data are collected with FD-NIRS instruments.

\section{Instrumentation for FD-NIRS}

A review of basic instrumentation aspects of FD-NIRS can be found in Chance et al. (1998b) and in Bigio and Fantini (2016), Section 13.4.2). A description of FD-NIRS instruments used in a variety of applications is beyond the scope of this review article, which specifically focuses on non-invasive brain measurements. The interested reader is referred to the description of FDNIRS instruments based on homodyne detection (Yang et al., 
1997; Ramanujam et al., 1998; Cheung et al., 2001; Yu et al., 2003), heterodyne detection (Fantini et al., 1995; Ramanujam et al., 1998; McBride et al., 2001; Nissilä et al., 2002, 2005), and on a radiofrequency network analyzer (Pham et al., 2000; Gulsen et al., 2006).

Recent developments in FD-NIRS instrumentation include implementations based on a compact frequency-sweeping circuit board (No et al., 2008), digital heterodyning (Arnesano et al., 2012), direct digital sampling (Roblyer et al., 2013; Zimmermann et al., 2016), frequency division multiplexing (Torjesen et al., 2017; Zhao et al., 2018), CMOS integrated circuitry (Sthalekar and Joyner Koomson, 2013; Yun and Joyner Koomson, 2013), and vertical cavity surface emitting lasers (VCSEL) (Sultan et al., 2013; Kitsmiller et al., 2018).

With regards to commercially available FD-NIRS instruments, they have only been made available by NIM, Inc. (Philadelphia, PA, United States), which operated from 1996 to 2009 (Ferrari and Quaresima, 2012), and by ISS, Inc. (Champaign, IL, United States), which introduced its first FD-NIRS instrument in the late 1990's and now offers FD-NIRS instruments for quantitative spectroscopy, oximetry, and imaging. Most noninvasive FD-NIRS studies of the brain are currently performed with ISS instruments, which operate by parallel multi-channel heterodyne detection and temporal multiplexing of light sources.

\section{Measurement of Absolute Optical Properties With FD-NIRS}

Diffusion theory yields analytical expressions of the FD-NIRS signals collected in a semi-infinite geometry. In this case, the light source and the optical detector are located on the tissue boundary, and the tissue is considered to be a uniform, semi-infinite medium, with optical properties described by an absorption coefficient $\left(\mu_{a}\right)$ and a reduced scattering coefficient $\left(\mu_{s}^{\prime}\right)$. Such analytical solutions show that one can define functions of measured DC, AC, and phase signals $\left(F_{D C}, F_{A C}, F_{\varphi}\right.$, respectively) that are linearly dependent on the distance $(r)$ between the source and the detector [as one may derive, for example, from Eqs. (12.33), (12.35), and (12.36) in Bigio and Fantini (2016; Section 12.6)], even though actual measurements of such functions of DC, AC, and phase are also affected by instrumental terms associated with the source and the detector:

$$
\begin{gathered}
F_{D C}\left(D C, \rho, \mu_{a}, \mu_{s}^{\prime}\right) \\
=S_{D C}\left(\mu_{a}, \mu_{s}^{\prime}\right) \rho+K_{D C-\text { Source }}+K_{D C-\text { Detector }} \\
F_{A C}\left(A C, \rho, \omega, \mu_{a}, \mu_{s}^{\prime}\right) \\
=S_{A C}\left(\omega, \mu_{a}, \mu_{s}^{\prime}\right) \rho+K_{A C-\text { Source }}+K_{A C-\text { Detector }} \\
F_{\varphi}\left(\varphi, \rho, \omega, \mu_{a}, \mu_{s}^{\prime}\right) \\
=S_{\varphi}\left(\omega, \mu_{a}, \mu_{s}^{\prime}\right) \rho+K_{\varphi-\text { Source }}+K_{\varphi-\text { Detector }}
\end{gathered}
$$

In Eqs. (2-4), $S_{D C}, S_{A C}, S_{\varphi}$ are the slopes of the linear functions of $r$, and the additive $K$ terms indicate instrumental factors contributed by the source or the detector to the DC, $\mathrm{AC}$, or phase measurement, as indicated by the subscripts. While the $K$ terms are unknown, as they are determined by several instrumental factors as well as by the optical coupling of the source and the detector with tissue, the functions $F$ and the slopes $S$ are known from diffusion theory. The functions $F$ can be approximated with $F_{D C}\left(D C, \rho, \mu_{a}, \mu_{s}^{\prime}\right)=\ln \left(\rho^{2} D C\right)$, $F_{A C}\left(A C, \rho, \mu_{a}, \mu_{s}^{\prime}\right)=\ln \left(\rho^{2} A C\right)$, and $F_{\varphi}\left(\varphi, \rho, \omega, \mu_{a}, \mu_{s}^{\prime}\right)=\varphi$ if the condition $\rho \sqrt{3 \mu_{a} \mu_{s}^{\prime}} \gg 1$ is fulfilled (Fantini et al., 1999). Equations (2-4) provide a basic description of how FD-NIRS can accomplish absolute measurements of optical properties according to different methods that are described below.

\section{Multi-Distance Methods}

Multi-distance methods rely on measurements at multiple (at least two) source-detector distances $(\rho)$, typically within a range of $1.5-4 \mathrm{~cm}$. The goal is to measure the slopes $\left[S_{D C}, S_{A C}, S_{\varphi}\right.$ in Eqs. (2-4)], which only depend on the optical properties (and the known modulation frequency) through analytical expressions (at least for homogeneous media) that can be inverted to yield absolute values of $\mu_{a}$ and $\mu_{s}^{\prime}$. Two data types (either DC and $\varphi$, or $\mathrm{AC}$ and $\varphi$ ) are needed to determine the two optical coefficients (Fantini et al., 1994a). The solution of the diffusion equation for semi-infinite media with extrapolated boundary conditions (Haskell et al., 1994) has been used in an iterative approach that accounts for the presence of $\mu_{a}$ and $\mu_{s}^{\prime}$ on the left-hand side of Eqs. (2-4) (Fantini et al., 1994b). As mentioned above, under the assumption $\rho \sqrt{3 \mu_{a} \mu_{s}^{\prime}} \gg 1$ one may use approximate expressions for $F_{D C}, F_{A C}$, and $F_{\varphi}$ (namely $\ln \left(\rho^{2} D C\right), \ln \left(\rho^{2} A C\right)$, and $\varphi)$ that offer the advantage that $\mu_{a}$ and $\mu_{s}^{\prime}$ do not appear on the left-hand side of Eqs. (2-4) (Fantini et al., 1999). We observe that in the case of non-invasive NIRS measurements of the brain (for which $\mu_{a}$ and $\mu_{s}^{\prime}$ are of the order of $0.1 \mathrm{~cm}^{-1}$ and $8 \mathrm{~cm}^{-1}$, respectively) the condition $\rho \sqrt{3 \mu_{a} \mu_{s}^{\prime}} \gg 1$ starts becoming fulfilled at source-detector distances $\rho$ of about $2 \mathrm{~cm}$, for which $\rho \sqrt{3 \mu_{a} \mu_{s}^{\prime}} \approx 2$, as we verified by comparing the results based on this approximation with those obtained with an iterative approach applied to the full solution of the diffusion equation.

Equations (2-4) clarify the importance of the sourcedetector configuration used in multi-distance methods. If the configuration uses a single detector and multiple sources placed at different distances from it, then the $K_{\text {Detector }}$ terms in Eqs. (2-4) are all independent of $\rho$ (i.e., they are the same for all sourcedetector pairs) and therefore they cancel out in the determination of the slopes $S$. By contrast, the $K_{\text {Source }}$ terms associated with each of the multiple sources are all different and must somehow be determined by a preliminary calibration on a tissue-like phantom with known optical properties. A reciprocal argument applies to the case of a configuration that uses a single source and multiple detectors (in which case, of course, it is the $K_{\text {Detector }}$ terms that are different for each source-detector pair). In a case 
where one uses a single source and a single detector, so that multidistance measurements are accomplished by scanning the source and/or detector relative to each other, no preliminary calibration is needed, since both $K_{\text {Source }}$ and $K_{\text {Detector }}$ terms are the same for all measurements at different values of $\rho$ (under the assumption that the optical coupling with tissue is kept constant throughout the scanning of source or detector). While this latter approach is the most robust for measurements in a lab setting, especially on tissue-like phantoms (Fantini et al., 1994b; Hallacoglu et al., 2013; Applegate and Roblyer, 2018), it is not practical for in vivo measurements, for which either multiple sources or multiple detectors are typically used. It is very important to observe that the multi-distance methods described above require that the optical coupling be the same in the calibration phantom and in tissue (for the method based on multiple sources or multiple detectors), or at the multiple source-detector distances considered (for the methods based on scanning the single source or single detector). This is an issue of high practical importance, and a potential limitation that can be addressed by the multidistance method described next.

An ingenious, yet simple multi-distance method for absolute measurements of optical properties that does not require any preliminary calibration and does not require assumptions about optical coupling with tissue is the self-calibrating method (Hueber et al., 1999). This method uses two sources and two detectors that are arranged in such a way to generate data described by two sets of Eqs. (2-4) where the $K_{\text {Source }}$ and $K_{\text {Detector }}$ terms have opposite signs, and thus can be canceled out by taking the average of the two sets of equations (Hueber et al., 1999). This method corrects for both instrumental and optical coupling contributions to the $K_{\text {Source }}$ and $K_{\text {Detector }}$ terms, thus minimizing potential confounding effects related to instrumental drifts, variable optical coupling with tissue, and motion artifacts.

\section{Single-Distance Methods}

One possible approach to absolute measurements with singledistance FD-NIRS data is to perform a preliminary calibration on a phantom with known optical properties to determine the term $K_{\text {Source }}+K_{\text {Detector }}$ in Eqs. $(2-4)$. While in principle this method is straightforward, it relies on a much more limiting assumption than the calibration methods for multi-distance measurements. While multi-distance calibrations assume that the relative optical coupling at the various distances is the same on the calibration phantom and on tissue, the single-distance calibration assumes that the absolute optical coupling is the same on phantom and tissue. This is a much stronger assumption that is difficult to realize in practice.

A more robust method for absolute single-distance measurements in the frequency domain is a multi-frequency approach. This method is also based on the solution of the diffusion equation represented by Eqs. (2-4). However, while in multi-distance methods the independent variable is the source-detector distance, $\rho$ (i.e., FD-NIRS data are measured at several source-detector distances), in the multi-frequency approach the independent variable is the modulation frequency, $\omega$ (i.e., FD-NIRS data are measured at several modulation frequencies). Usually the modulation frequency is swept from tens to hundreds of $\mathrm{MHz}$, while the source-detector distance is fixed at about $3 \mathrm{~cm}$ (Pham et al., 2000; Gulsen et al., 2006). Of course, any dependence on $\omega$ of the $K_{\text {Source }}$ and $K_{\text {Detector }}$ terms must be considered through calibration measurements on a reference phantom or at multiple source-detector distances (Bevilacqua et al., 2000; Pham et al., 2000). Iterative methods based on non-linear least-squares fits have been used for the recovery of the optical properties in the multi-frequency approach (Haskell et al., 1994).

\section{Measurement of Absorption Changes With FD-NIRS}

Most non-invasive optical studies of the brain aim to detect absorption changes related to cerebral hemodynamic and oxygenation changes. One possible exception is the measurement of the fast optical signal, which will be presented in Section 3.6. In the case of a homogeneous absorption change, $\Delta \mu_{a}$, in a semiinfinite medium, one can translate relative changes in the DC Intensity $\left(\triangle I_{D C} / I_{D C 0}\right.$, where we indicate with $I_{D C 0}$ the detected DC intensity in a reference condition, say at baseline, and with $\Delta I_{D C}$ the intensity change with respect to the reference condition as a result of an absorption change $\Delta \mu_{a}$ ) and absolute changes in the phase $(\Delta \varphi)$ into the corresponding absorption change as follows (Blaney et al., 2020; Fantini et al., 2020):

$$
\begin{gathered}
\Delta \mu_{a}\left(I_{D C}\right)=-\frac{2\left(1+\rho \sqrt{3 \mu_{a 0} \mu_{s 0}^{\prime}}\right)}{3 \rho^{2} \mu_{s 0}^{\prime}}\left(\frac{\Delta I_{D C}}{I_{D C 0}}\right) \\
\Delta \mu_{a}(\varphi)=-\left(\frac{1+\frac{\sqrt{\gamma+1}}{\gamma \rho} \sqrt{\frac{2}{3 \mu_{a 0} \mu_{s 0}^{\prime}}}+\frac{1}{3 \mu_{a 0} \mu_{s 0}^{\prime} \gamma \rho^{2}}}{\rho \frac{\sqrt{\gamma-1}}{2 \gamma} \sqrt{\frac{3 \mu_{s 0}^{\prime}}{2 \mu_{a 0}}}}\right) \Delta \varphi
\end{gathered}
$$

where the subscripts 0 indicate a reference condition for which $\mu_{a}=\mu_{a 0}$, the changes with respect to this reference condition are $\Delta \mu_{a}=\mu_{a}-\mu_{a 0}, \Delta I_{D C}=I_{D C}-I_{D C 0}, \Delta \varphi=\varphi-\varphi_{0}$, and $\gamma=\sqrt{1+\left(\frac{\omega}{c_{n} \mu_{a 0}}\right)^{2}}$, with $c_{n}$ speed of light in tissue. Equation (5) represents the modified Beer-Lambert law that is extensively used in CW-NIRS (Delpy et al., 1988; Sassaroli and Fantini, 2004). A correction to Eq. (5) is needed for the AC amplitude of intensity $\left(I_{A C}\right)$; such correction can be found in Sassaroli et al. (2019) and Blaney et al. (2020). The effect of localized absorption changes on the DC Intensity (and similarly on the AC amplitude) and on the phase can be described in terms of spatial regions of sensitivity, which are introduced in Section "The Spatial Region of Sensitivity of DC, AC, and Phase."

In the case of high modulation frequencies, such that $\omega \gg$ $c_{n} \mu_{a}$, one can show that the term in parenthesis on the right-hand side of Eq. (6) becomes independent of $\mu_{a 0}$ so that, under the further assumption that scattering is independent on wavelength, the ratio of phase changes at two wavelengths $\lambda_{1}$ and $\lambda_{2}$ equals the ratio of absorption changes at the same wavelengths:

$$
\frac{\Delta \varphi\left(\lambda_{1}\right)}{\Delta \varphi\left(\lambda_{2}\right)}=\frac{\Delta \mu_{a}\left(\varphi, \lambda_{1}\right)}{\Delta \mu_{a}\left(\varphi, \lambda_{2}\right)}
$$


In the ideal case of a baseline condition that features no absorption (i.e., $\mu_{a 0}=0$ ), $\Delta \mu_{a}=\mu_{a}$ and one can write (Sevick et al., 1991):

$$
\frac{\Delta \varphi\left(\lambda_{1}\right)}{\Delta \varphi\left(\lambda_{2}\right)}=\frac{\mu_{a}\left(\varphi, \lambda_{1}\right)}{\mu_{a}\left(\varphi, \lambda_{2}\right)}
$$

This result may be used to quantify the oxygen saturation of hemoglobin associated with an absorption change from baseline, but the condition $\omega \gg c_{n} \mu_{a}$ and the assumption $\mu_{s 0}^{\prime}\left(\lambda_{1}\right) \cong$ $\mu_{s 0}^{\prime}\left(\lambda_{2}\right)$ are somewhat limiting. In fact, for $\mu_{a}=0.05 \mathrm{~cm}^{-1}$, one would need to use a modulation frequency $[f=\omega /(2 \pi)]$ that is much greater than $\sim 170 \mathrm{MHz}$.

\section{The Spatial Region of Sensitivity of DC, $A C$, and Phase}

In this section, we describe the spatial region in tissue that is probed in FD-NIRS by a given source-detector pair that is placed on the tissue surface. This description requires several specifications. In fact, (1) different data types (DC, AC, $\varphi$ ) have different regions of sensitivity; (2) the sensitivity itself is not constant within the overall probed volume, so that a given optical perturbation in some portions of the probed region induce stronger changes in the optical signals than others; (3) the region of sensitivity can be different for different kinds of optical contrast, say for an absorption vs. a scattering perturbation. Here, we consider localized changes within a small region (conceptually a point-like region), in either the absorption coefficient or reduced scattering coefficient, in an otherwise optically homogeneous medium. The case of an absorption perturbation has been studied in two opposite regimes: in the limit of infinitely absorbing defects (Feng et al., 1995) and in the limit of infinitesimal changes in the absorption coefficient (Born approximation) (Boas, 1997). The relationship between these two extreme approaches has been recently investigated (Sassaroli et al., 2014). For the case of a scattering perturbation, only the Born approximation has been considered (Martelli et al., 2010). The region of sensitivity for a specific optical measurement with respect to a local change in optical properties also depends on the background optical properties, the shape of tissue and the nature of boundary conditions, and the spatial arrangements of source(s) and detector(s). If we denote with $Y$ any optical measurement (for example, DC, AC, or phase in FD-NIRS), we define a dimensionless sensitivity of such a measurement to a point-like perturbation in a given optical coefficient $\mu$ (which may be either $\mu_{a}$ or $\mu_{s}^{\prime}$ ) as follows:

$$
S_{Y, \mu}(\mathbf{r})=\frac{\left(\frac{\partial Y}{\partial \mu_{\mathbf{r}}}\right)}{\left(\frac{\partial Y}{\partial \mu}\right)}
$$

where the derivative in the numerator is taken with respect to a point-like perturbation at position $\mathbf{r}\left(\mu_{\mathbf{r}}\right)$, whereas the derivative in the denominator is taken with respect to a uniform change, everywhere in the medium, in the optical property $\mu$.

We have calculated the spatial sensitivity defined in Eq. (9) using diffusion theory for a semi-infinite medium of optical properties $\mu_{a}=0.1 \mathrm{~cm}^{-1}$ and $\mu_{s}^{\prime}=10 \mathrm{~cm}^{-1}$, a single sourcedetector pair placed on the medium boundary at a sourcedetector distance of $35 \mathrm{~mm}$, and an optical perturbation $1 \mathrm{~mm}^{3}$ in size. The modulation frequency was $140 \mathrm{MHz}$. The results are reported in Figure $\mathbf{1}$ for DC intensity (top panels) and phase (bottom panels), and for perturbations in absorption (left panels) or scattering (right panels). Positive sensitivity values at any position $\mathbf{r}$ mean that the considered data changes in the same direction (increase or decrease) as a result of a localized change at $\mathbf{r}$ or a homogeneous change in the considered optical property. Figure 1 shows that the DC intensity sensitivity (i.e., the sensitivity in CW-NIRS) is always positive, for both absorption and scattering perturbations (with a possible exception of scattering defects near the source or the detector), and has a typical banana shape (Feng et al., 1995). Also, DC intensity sensitivity is especially high for optical perturbations close to the source and the detector. The phase sensitivity is more complex. In the case of absorption perturbations, it features a "double banana, a shallower one of negative sensitivity and a deeper one of positive sensitivity. In the case of scattering perturbations, the phase sensitivity is mostly positive, and also banana shaped, except for small regions close to the source and the detector. Based on Figure 1, one can appreciate the different nature of the optical sensitivity of intensity and phase data to absorption and scattering contrast.

\section{USE OF FD-NIRS FOR NON-INVASIVE BRAIN STUDIES}

\section{Imaging With Phased Arrays}

In Section "The FD-NIRS Data: DC Intensity, AC Amplitude, Phase," we mentioned that the photon-density waves generated by the intensity-modulated light sources in FD-NIRS are nearfield waves. They are only measurable within a fraction of a wavelength. This is in contrast with the case of ultrasound imaging, where the ultrasound wavelength in soft tissue is typically of the order of hundreds of microns, and phased arrays are commonly used to focus and steer ultrasound beams. Nevertheless, phased arrays have also been proposed in FD-NIRS to exploit the interference of photon-density waves (Schmitt et al., 1992). In particular, it was proposed to achieve the accurate localization of small defects by scanning the nullplane generated by two light sources that are modulated in opposition of phase (Chance et al., 1993; Chance et al., 1996). This method has been applied to the study of sensorimotor (finger touching) and cognitive (backward spelling) activation in human subjects, demonstrating a high signal-to-noise ratio $(>40)$ of phase measurements, resulting from the large (180 degrees) phase transition at the null plane (Chance et al., 1998a). The phased-array method has been used as a basis for diffuse optical tomography (Intes et al., 2002; Rajan et al., 2008), and it was proposed to implement it by post-processing CW-NIRS data (Liu et al., 2005a,b). The emphasis of the phased array approach is on an improvement of the spatial localization and resolution in diffuse optics. Because such an improvement comes 

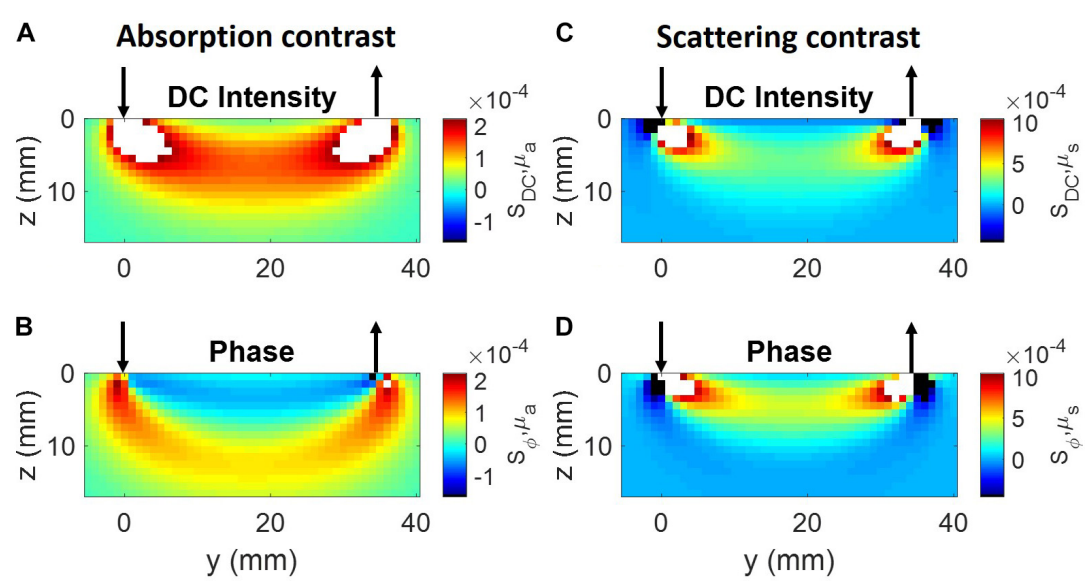

FIGURE 1 | Sensitivity maps in the ( $y . z)$ plane, where the tissue boundary is the $x-y$ plane, the source (arrow pointing down) is at $(0,0,0)$, and the detector (arrow pointing up) is at (0, $35 \mathrm{~mm}, 0)$. (A) DC Intensity, absorption contrast; (B) phase, absorption contrast; (C) DC intensity, scattering contrast, (D) phase, scattering contrast. The color bar labels in panels $(\mathbf{A}, \mathbf{C})$ indicate the sensitivity of DC intensity with respect to absorption $\left(S_{D C, \mu_{a}}\right)$ and reduced scattering coefficients $\left(S_{D C, \mu_{s}}\right)$, respectively. The color bar labels in panels $(\mathbf{B}, \mathbf{D})$ indicate the sensitivity of phase with respect to absorption $\left(S_{\phi, \mu_{a}}\right)$ and reduced scattering coefficients $\left(S_{\phi, \mu_{s}}\right)$, respectively. White and black in the color maps indicate values greater than the maximum or smaller than the minimum, respectively, of the color bars.

at the expense of a more complex instrumentation, targets specific classes of dynamic perturbations, and introduces some limitations to the field of view, it has not found broad applicability in diffuse optics. Of course, this does not mean that it may not be implemented in such a way to benefit specific applications in the near future.

\section{Absolute Measurements With a Combination of Intensity and Phase Data}

A common use of time-resolved NIRS, in either the time domain or the frequency domain, is toward measurements of absolute optical properties of tissue, namely its absorption $\left(\mu_{a}\right)$ and reduced scattering $\left(\mu_{s}^{\prime}\right)$ coefficients. Measurements at a minimum of two wavelengths, in conjunction with the Beer's law relationship between absorption and chromophores concentration, allow for the translation of absorption coefficients into cerebral concentrations of oxy-hemoglobin $\left(\left[\mathrm{HbO}_{2}\right]\right)$, deoxy-hemoglobin $([\mathrm{Hb}])$, total hemoglobin $\left([\mathrm{HbT}]=\left[\mathrm{HbO}_{2}\right]+[\mathrm{Hb}]\right)$, and hemoglobin saturation $\left(\mathrm{StO}_{2}=\left[\mathrm{HbO}_{2}\right] /[\mathrm{HbT}]\right)$. Here, the " $\mathrm{t}$ " in $\mathrm{StO}_{2}$ indicates that the oxygen saturation of hemoglobin refers to an average over the investigated tissue. It is important to note that absolute measurements of $\mathrm{StO}_{2}$ may still be obtained from absorption coefficients that are only known to within an unknown factor, because $\mathrm{StO}_{2}$ depends on the ratio of absorption coefficients at multiple wavelengths (Bigio and Fantini, 2016, Section 15.1.3.1). This fact accounts for more robust absolute measurements of $\mathrm{StO}_{2}$ with respect to $[\mathrm{Hb}]$ or $\left[\mathrm{HbO}_{2}\right]$, which instead rely on accurate absolute measurements of $\mu_{a}$. For this reason, it is possible that $\mathrm{FD}$ and $\mathrm{CW}$ measurements of $\mathrm{StO}_{2}$ (where the latter typically need to introduce some ad hoc assumptions on the scattering properties of tissue) may yield comparable results, as recently reported in a study on healthy human subjects during controlled hypoxia (Davies et al., 2017).
Multi-distance methods (see Section "Multi-Distance Methods") have been employed for absolute measurements of cerebral concentration and/or saturation of hemoglobin in newborn piglets (Fantini et al., 1999; Zhang et al., 2000), in rats (Culver et al., 2003), in newborn and infants (Franceschini et al., 2007; Grant et al., 2009; Roche-Labarbe et al., 2010, 2012; Lin et al., 2013b, Lin T.-Y. et al., 2016; Dehaes et al., 2014, 2015; Demel et al., 2014b, 2015; Farzam et al., 2017; Ferradal et al., 2017; Schwarz et al., 2018), and in adults under normal conditions (Fantini et al., 2003; Gatto et al., 2006, 2007; McIntosh et al., 2010; Hallacoglu et al., 2012, 2013; Scholkmann et al., 2013a; Clancy et al., 2015; Kainerstorfer et al., 2015; Yang and Dunn, 2015; Moreau et al., 2016; Davies et al., 2017; Blaney et al., 2019; Pham et al., 2019), under anesthesia (Paisansathan et al., 2007; Meng et al., 2012a,b), hypoxia (Davies et al., 2017), pathological conditions such as multiple sclerosis (Yang and Dunn, 2015), stroke (Moreau et al., 2016), traumatic brain injury (Davies et al., 2019), before and during electro-convulsive therapy (ECT) (Fabbri et al., 2003), during neurovascular surgery (Calderon-Arnulphi et al., 2007), and after death (Gatto et al., 2006). The absolute values of $[\mathrm{Hb}],\left[\mathrm{HbO}_{2}\right],[\mathrm{HbT}]$, and $\mathrm{StO}_{2}$ reported in these studies are summarized in Table $\mathbf{1}$ for piglets and rats, Table 2 for neonates and infants, and Table 3 for human adults. The absolute values of hemoglobin concentration and saturation reported in Tables $\mathbf{1 - 3}$ represent typical reported values (thus no error is provided) under baseline conditions at rest (unless otherwise noted).

Some studies have also reported the optical coefficients $\left(\mu_{a}\right.$ and $\mu_{s}^{\prime}$ ) of brain tissue at discrete FD-NIRS wavelengths. This was done in newborn piglets (Fantini et al., 1999; Zhang et al., 2000), in neonates and infants (Zhao et al., 2005; Demel et al., 2014a; Farzam et al., 2017; Tian et al., 2017), and in adults (Hallacoglu et al., 2012; Blaney et al., 2019). Typically, optical properties were obtained by considering the tissue as homogeneous. However, a two-layer model has also been applied to analyze FD-NIRS 


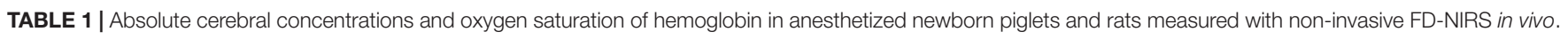

\begin{tabular}{|c|c|c|c|c|c|c|c|c|c|c|}
\hline References & Instr. & $f(\mathrm{MHz})$ & Method & $\#$ of $\lambda$ & {$[\mathrm{Hb}](\mu \mathrm{M})$} & {$\left[\mathrm{HbO}_{2}\right](\mu \mathrm{M})$} & {$[\mathrm{HbT}](\mu \mathrm{M})$} & $\mathrm{StO}_{2}(\%)$ & Age (days) & Animal model \\
\hline Ntziachristos et al., 1997 & NIM & 200 & $\mathrm{SD}$ (phase): $\sim 2 \mathrm{~cm}$ & 2 & - & - & - & 80 & $1-5$ & Piglet \\
\hline Fantini et al., 1999 & ISS & 110 & MD: $1.5-3 \mathrm{~cm}$ & 2 & 17 & 26 & 43 & 60 & $11 \pm 1$ & Piglet \\
\hline Zhang et al., 2000 & ISS & 110 & MD: $1.5-3 \mathrm{~cm}$ & 2 & 15 & 30 & 45 & 67 & $9 \pm 2$ & Piglet \\
\hline Culver et al., 2003 & Own & 70 & MD: $0.3-1 \mathrm{~cm}$ & 3 & 30 & 70 & 100 & 70 & Adult & Rat \\
\hline
\end{tabular}

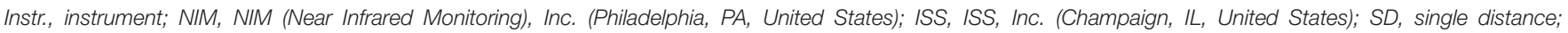
MD, multi distance.

TABLE 2 | Absolute cerebral concentrations and oxygen saturation of hemoglobin in neonates, infants, and children measured with non-invasive FD-NIRS in vivo.

\begin{tabular}{|c|c|c|c|c|c|c|c|c|c|}
\hline References & Instr. & $f(\mathrm{MHz})$ & Method & $\#$ of $\lambda$ & [Hb] $(\mu \mathrm{M})$ & {$\left[\mathrm{HbO}_{2}\right](\mu \mathrm{M})$} & {$[\mathrm{HbT}](\mu \mathrm{M})$} & $\mathrm{StO}_{2}(\%)$ & Age \\
\hline Watzman et al., 2000 & NIM & 200 & SD: 3 or $4 \mathrm{~cm}$ & 3 & - & - & - & 73 & $0-6$ years \\
\hline Zhao et al., 2005 & Own & 140 & SD: $4 \mathrm{~cm}$ & 2 & 16 & 24 & 40 & 59 & $1-17$ days \\
\hline \multirow[t]{4}{*}{ Franceschini et al., 2007} & ISS & 110 & MD: $1-2.5 \mathrm{~cm}$ & 7 & 17 & 35 & 52 & 67 & 1 week \\
\hline & & & & & 14 & 19 & 33 & 57 & 6 weeks \\
\hline & & & & & 21 & 32 & 53 & 61 & 12 weeks \\
\hline & & & & & 24 & 49 & 73 & 67 & 52 weeks \\
\hline Grant et al., 2009 & ISS & 110 & MD: $1-2.5 \mathrm{~cm}$ & 7 & 21 & 39 & 60 & 65 & 0-15 days \\
\hline \multirow[t]{2}{*}{ Roche-Labarbe et al., 2010} & ISS & 110 & MD: $1-2.5 \mathrm{~cm}$ & 6 & 12 & 28 & 40 & 70 & 1 week \\
\hline & & & & & 12 & 18 & 30 & 60 & 6 weeks \\
\hline \multirow[t]{2}{*}{ Roche-Labarbe et al., 2012} & ISS & 110 & MD: $1-2.5 \mathrm{~cm}$ & 6 & 15 & 40 & 55 & 73 & 1 week \\
\hline & & & & & 15 & 28 & 43 & 65 & 10 weeks \\
\hline Lin et al., 2013b & ISS & 110 & MD: $1-2.5$ or $1.5-3 \mathrm{~cm}$ & 6 & 19 & 41 & 60 & 69 & $3.6 \pm 1.7$ weeks \\
\hline Dehaes et al., 2014 & ISS & 110 & MD: $1-2.5 \mathrm{~cm}$ & 6 & 17 & 45 & 62 & 72 & $0-4$ days \\
\hline Demel et al., 2014b & ISS & 110 & MD: $1.5-3 \mathrm{~cm}$ & 2 & 15 & 23 & 38 & 60 & 2 days \\
\hline Dehaes et al., 2015 & ISS & 110 & MD: $1.5-3 \mathrm{~cm}$ & 6 & 16 & 35 & 51 & 69 & $33-71 \mathrm{~h}$ \\
\hline \multirow[t]{2}{*}{ Demel et al., 2015} & ISS & 110 & MD: $1.5-3 \mathrm{~cm}$ & 2 & 15 & 24 & 39 & 62 & 7-71 h (preterm) \\
\hline & & & & & 18 & 30 & 48 & 63 & 7-54 h (term) \\
\hline Lin P. Y. et al., 2016 & ISS & 110 & MD: 1-2.5 cm & 6 & - & - & - & $50-70$ & 3-14 weeks (preterm) \\
\hline \multirow[t]{2}{*}{ Farzam et al., 2017} & ISS & 110 & MD: $1.5-3 \mathrm{~cm}$ & 8 & 19 & 41 & 60 & 69 & $2.4 \pm 0.8$ days (males) \\
\hline & & & & & 16 & 33 & 50 & 65 & $2.6 \pm 1.5$ days (females) \\
\hline Ferradal et al., 2017 & ISS & 110 & MD: $1.5-3 \mathrm{~cm}$ & 8 & 26 & 40 & 66 & 60 & $\sim 4$ days (anesthesia) \\
\hline Schwarz et al., 2018 & ISS & 110 & MD: $1.5-3 \mathrm{~cm}$ & 2 & - & - & - & 54 & 4-13 days \\
\hline
\end{tabular}

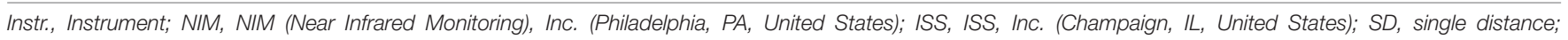
MD, multi distance.

data collected on adult human subjects to separately measure the optical properties of the superficial, extracerebral tissue (top layer) and the deeper brain tissue (bottom layer) (Hallacoglu et al., 2013). The absolute values of $\mu_{a}$ and $\mu_{s}^{\prime}$ reported in these studies are summarized in Table 4 and represent typical values (no error is provided) under baseline conditions.

The optical properties measured with multi-distance FDNIRS on the forehead of sixteen elderly subjects ( $85 \pm 6$ years old) by Hallacoglu et al. (2012) are reported in Figure 2 for different ranges of source-detector distance using diffusion theory for a homogeneous semi-infinite medium. Optical measurements were taken on the same group of subjects in two separate sessions separated in time by 5 months. The two measurement sessions are represented in Figure 2 by gray and black blocks, respectively. The source-detector distances employed in the first session were $2,2.5,3$, and $3.5 \mathrm{~cm}$, whereas in the second session they were $0.8,1.3,1.8,2.3,2.8,3.3$, and $3.8 \mathrm{~cm}$. The horizontal extent of the blocks in Figure 2 indicates the distance range used for measurements of the optical properties according to the multi-distance method of Section "Multi-Distance Methods" (Fantini et al., 1999). The vertical extent of the blocks in Figure 2 represent the mean value \pm the standard error of measurements on different subjects. Because data collected at longer distances are more sensitive to deeper tissue, the strong decrease in scattering vs. distance (i.e., the lower scattering values associated with the blocks representing measurements at longer source-detector distances in the middle panels of Figure 2) suggests that brain tissue may be less scattering than superficial scalp and skull tissue, even though the low scattering of the cerebrospinal fluid layer may also contribute to this result. This finding of a lower scattering of deeper tissue (subarachnoid space, brain, etc.) vs. superficial tissue (scalp, skull, etc.) is confirmed by a study that considered diffusion theory for a two-layered medium, and found a significantly lower scattering coefficient in the bottom tissue layer compared to the top tissue layer, whose thickness was found to be about $13 \mathrm{~mm}$ (Hallacoglu et al., 2013) (see Table 4). 
TABLE 3 | Absolute cerebral concentrations and oxygen saturation of hemoglobin in human adults measured with non-invasive FD-NIRS in vivo.

\begin{tabular}{|c|c|c|c|c|c|c|c|c|c|c|}
\hline References & Instr. & $f(\mathrm{MHz})$ & Method & $\#$ of $\lambda$ & {$[\mathrm{Hb}](\mu \mathrm{M})$} & {$\left[\mathrm{HbO}_{2}\right](\mu \mathrm{M})$} & {$[\mathrm{HbT}](\mu \mathrm{M})$} & $\mathrm{StO}_{2}(\%)$ & Age (y) & Notes \\
\hline Fabbri et al., 2003 & ISS & 110 & MD: $1.5-3$ or $1-2.5 \mathrm{~cm}$ & 2 & 14 & 19 & 33 & 57 & $27-57$ & Before ECT \\
\hline Fantini et al., 2003 & ISS & 110 & MD: & 2 & 14 & 20 & 34 & 59 & $29-44$ & Before sleep \\
\hline Gatto et al., 2006 & ISS & 110 & MD: $2-4 \mathrm{~cm}$ & 2 & 13 & 24 & 37 & 64 & $36 \pm 9$ & \\
\hline \multirow[t]{2}{*}{ Gatto et al., 2007} & ISS & 110 & MD: 2-4 cm & 2 & 15 & 28 & 43 & 65 & 20-39 & \\
\hline & & & & & 13 & 22 & 35 & 63 & $40-60$ & \\
\hline Calderon-Arnulphi et al., 2007 & ISS & 110 & MD: $2-3.5 \mathrm{~cm}$ & 2 & 16 & 16 & 32 & 50 & $58-81$ & Before surgery \\
\hline Paisansathan et al., 2007 & ISS & 110 & MD: $2-4 \mathrm{~cm}$ & 2 & 13 & 19 & 32 & 59 & $44 \pm 14$ & Anesth. \\
\hline Mclntosh et al., 2010 & ISS & 110 & MD: $2-3.5 \mathrm{~cm}$ & 2 & 19 & 26 & 45 & 58 & $20-53$ & \\
\hline Meng et al., 2012a & ISS & 110 & MD: $2-3.5 \mathrm{~cm}$ & 2 & 11 & 30 & 41 & 73 & $22-68$ & Anesth. \\
\hline Meng et al., 2012b & ISS & 110 & MD: $2-3.5 \mathrm{~cm}$ & 2 & 11 & 25 & 36 & 68 & $22-68$ & Anesth. \\
\hline \multirow[t]{2}{*}{ Hallacoglu et al., 2012} & ISS & 110 & MD: $2.8-3.8 \mathrm{~cm}$ & 2 & 20 & 39 & 59 & 66 & $28 \pm 4$ & \\
\hline & & & & & 18 & 22 & 40 & 55 & $85 \pm 6$ & \\
\hline \multirow[t]{3}{*}{ Hallacoglu et al., 2013} & ISS & 110 & MD: $1.3-4.8 \mathrm{~cm}$ & 2 & 11 & 15 & 26 & 42 & $29 \pm 2$ & Superf. tissue \\
\hline & & & & & 31 & 51 & 82 & 64 & & Deep tissue \\
\hline & & & & & 17 & 30 & 47 & 64 & & Homog. tissue \\
\hline Scholkmann et al., 2013a & ISS & 110 & MD: $2-4 \mathrm{~cm}$ & 2 & - & - & - & 59 & - & \\
\hline Clancy et al., 2015 & ISS & 110 & $\mathrm{MD}$ & 2 & 11 & 29 & 40 & 72 & - & \\
\hline Yang and Dunn, 2015 & ISS & 110 & MD: 2-3.5 cm & 2 & 17 & 28 & 45 & 63 & $50 \pm 8$ & \\
\hline Kainerstorfer et al., 2015 & ISS & 110 & MD: $2-3.5 \mathrm{~cm}$ & 2 & 16 & 35 & 51 & 69 & $21-50$ & \\
\hline Moreau et al., 2016 & ISS & 110 & MD: 2-3.5 cm & 2 & 20 & 35 & 56 & 63 & $42 \pm 7$ & \\
\hline Davies et al., 2017 & ISS & 110 & $\mathrm{MD}$ & 2 & - & - & - & 62 & - & \\
\hline Davies et al., 2019 & ISS & 110 & MD: $3-4.5 \mathrm{~cm}$ & 2 & & & & 67 & $50 \pm 17$ & TBI \\
\hline Blaney et al., 2019 & ISS & 140 & MD: $1.1-4 \mathrm{~cm}$ & 2 & 18 & 24 & 42 & 56 & $25-53$ & \\
\hline Pham et al., 2019 & ISS & 140 & MD: $1.6-4 \mathrm{~cm}$ & 2 & 11 & 39 & 50 & 78 & $22-33$ & \\
\hline
\end{tabular}

Measurements are for healthy subjects at rest and based on diffusion theory for homogeneous tissue, unless otherwise noted in the "Notes" column. Instr., Instrument; ISS, ISS, Inc. (Champaign, IL, United States); MD, multi distance; ECT, electro-convulsive therapy; TBI, Traumatic Brain Injury.

In some cases, absolute measurements of baseline optical properties and hemoglobin concentrations obtained with multidistance FD-NIRS are not reported, as the focus was the investigation of quantitative changes in $[\mathrm{Hb}]$ and $\left[\mathrm{HbO}_{2}\right]$ associated with visual cortical activation in younger and older adults (McKernan Ward et al., 2015), brain activation with colored light (Scholkmann et al., 2017; Metz et al., 2017), a speech task (Scholkmann et al., 2013b, 2014a), or changes in cerebral blood flow (CBF) and $\mathrm{StO}_{2}$ following intrathoracic pressure strains (Zhang et al., 2017). Even in cases like these, where the emphasis is on measuring relative rather than absolute concentrations of hemoglobin, the ability of FD-NIRS to provide absolute measures of baseline optical properties can be beneficial. In fact, one does not need to rely on assumptions associated with the modified Beer-Lambert law in CW-NIRS, namely the values of the differential pathlength factor (DPF) at the wavelengths used (since the DPF can be obtained from the absolute optical properties), and the lack of changes in the scattering properties of tissue (since absolute scattering measurements allows one to verify whether scattering stays constant).

The absolute measurements of [HbT] (units: mol/l) afforded by FD-NIRS can be translated into absolute measurements of cerebral blood volume (CBV) [units: $\mathrm{ml} /(100 \mathrm{~g})$ ] by taking into account the concentration of hemoglobin in large blood vessels (HGB) (units: $\mathrm{mol} / \mathrm{l}$ ), the molecular weight of hemoglobin $\left(\mathrm{MW}_{\mathrm{Hb}}\right)$ (units: $\mathrm{mol} / \mathrm{g}$ ), the density of brain tissue $\left(D_{b t}\right)$ (units: $\mathrm{g} / \mathrm{ml}$ ), and the small-to-large vessel hematocrit ratio, or Fårhaeus factor $(F)$ (Franceschini et al., 2007; Meng et al., 2012a):

$$
C B V=\frac{[H b T] \times M W_{H b}}{H G B \times D_{b t} \times F}
$$

Absolute measurements of $\mathrm{StO}_{2}$ (dimensionless) with multidistance FD-NIRS have been combined with measurements of a cerebral blood flow index (BFI) with diffuse correlation spectroscopy (DCS) to yield a relative cerebral metabolic rate of oxygen $\left(\mathrm{rCMRO}_{2}\right)$, with respect to a reference condition indicated by 0 subscripts (Roche-Labarbe et al., 2012):

$$
r C M R O_{2}=\frac{H G B \times B F I \times\left(\mathrm{SaO}_{2}-\mathrm{StO}_{2}\right)}{H G B_{0} \times B F I_{0} \times\left(\mathrm{SaO}_{2}-\mathrm{StO}_{2}\right)_{0}}
$$

where $\mathrm{SaO}_{2}$ is the oxygen saturation of hemoglobin in arterial blood, and under the assumption of a constant relative contribution of arterial and venous blood to the $\mathrm{StO}_{2}$ measurement.

Using the approach of Eqs. (10) and (11), researchers have investigated the cerebral oxygen metabolism in neonates with intraventricular hemorrhage (Lin P. Y. et al., 2016) and the blood volume and metabolic responses to hand tactile stimulation in the somatosensory cortex of preterm neonates (Roche-Labarbe et al., 2014).

Single-distance methods (see Section "Single-Distance Methods") can also yield absolute measurements, as long as 
TABLE 4 | Absolute cerebral absorption and reduced scattering coefficients measured with non-invasive FD-NIRS in vivo.

\begin{tabular}{|c|c|c|c|c|c|c|c|c|}
\hline References & Instr. & $f(\mathrm{MHz})$ & Method & $\lambda(\mathrm{nm})$ & $\mu_{a}\left(\mathrm{~cm}^{-1}\right)$ & $\mu_{s}^{\prime}\left(\mathrm{cm}^{-1}\right)$ & Age & Brain investigated \\
\hline \multirow[t]{2}{*}{ Fantini et al., 1999} & ISS & 110 & MD: $1.5-3 \mathrm{~cm}$ & 758 & 0.15 & 9.3 & $11 \pm 1$ days & Piglets \\
\hline & & & & 830 & 0.13 & 8.2 & & \\
\hline \multirow[t]{2}{*}{ Zhang et al., 2000} & ISS & 110 & MD: $1.5-3 \mathrm{~cm}$ & 758 & - & 7.8 & $9 \pm 2$ days & Piglets \\
\hline & & & & 830 & - & 6.6 & & \\
\hline \multirow[t]{2}{*}{ Zhao et al., 2005} & Own & 140 & SD(calib): 4 cm & 788 & 0.078 & 9.2 & 1-17 days & Neonates/infants/children \\
\hline & & & & 832 & 0.089 & 8.4 & & \\
\hline \multirow[t]{2}{*}{ Demel et al., 2014a } & ISS & 110 & MD: $1.5-3 \mathrm{~cm}$ & 692 & $0.07-0.11$ & $5.9-9.4$ & 1-71 days & Neonates/infants/children \\
\hline & & & & 834 & $0.08-0.12$ & $4.5-7.2$ & & \\
\hline \multirow[t]{8}{*}{ Farzam et al., 2017} & ISS & 110 & MD: $1.5-3 \mathrm{~cm}$ & 672 & 0.160 & 8.7 & $2 \pm 1$ days & Neonates/infants/children \\
\hline & & & & 689 & 0.127 & 8.5 & & \\
\hline & & & & 701 & 0.115 & 8.5 & & \\
\hline & & & & 724 & 0.110 & 8.2 & & \\
\hline & & & & 771 & 0.140 & 7.5 & & \\
\hline & & & & 783 & 0.126 & 7.2 & & \\
\hline & & & & 803 & 0.121 & 6.8 & & \\
\hline & & & & 829 & 0.141 & 6.5 & & \\
\hline Tian et al., 2017 & ISS & 110 & MD & 785,811 & $0.15-0.30$ & $6-18$ & $0-16$ years $(\mathrm{ECMO})$ & Neonates/infants/children \\
\hline \multirow[t]{4}{*}{ Hallacoglu et al., 2012} & ISS & 110 & MD: $2.8-3.8 \mathrm{~cm}$ & 690 & 0.14 & 7.2 & $28 \pm 4$ years & Adults (homogeneous tissue) \\
\hline & & & & 830 & 0.12 & 6.0 & & \\
\hline & & & & 690 & 0.10 & 5.3 & $85 \pm 6$ years & \\
\hline & & & & 830 & 0.08 & 4.8 & & \\
\hline \multirow[t]{6}{*}{ Hallacoglu et al., 2013} & ISS & 110 & MD: $1.3-4.8 \mathrm{~cm}$ & 690 & 0.08 & 13 & $29 \pm 2$ years & Adults (superficial tissue, $\sim 13 \mathrm{~mm}$ ) \\
\hline & & & & 830 & 0.08 & 11 & & \\
\hline & & & & 690 & 0.20 & 3 & & Adults (deep tissue, brain) \\
\hline & & & & 830 & 0.20 & 2 & & \\
\hline & & & & 690 & 0.11 & 7 & & Adults (homogeneous tissue) \\
\hline & & & & 830 & 0.12 & 5 & & \\
\hline \multirow[t]{2}{*}{ Blaney et al., 2019} & ISS & 140 & MD: $1.1-4 \mathrm{~cm}$ & 690 & 0.10 & 9.8 & $25-53$ years & Adults (homogeneous tissue) \\
\hline & & & & 830 & 0.11 & 8.0 & & \\
\hline
\end{tabular}

Instr., Instrument; ISS, ISS, Inc. (Champaign, IL, United States); MD, multi distance; SD, single distance; ECMO, Extracorporeal Membrane Oxygenation.

they include a suitable calibration on a reference phantom with known optical properties. This method has been applied to the infant brain (Zhao et al., 2005).

\section{Stand-Alone Use of DC Data}

There are research studies in the literature that are performed with FD-NIRS instrumentation, but report results obtained exclusively with DC intensity, which is equivalent to what would be measured in CW-NIRS. For example, this is the case for studies on the cerebral hemodynamics associated with auditory stimulation [concurrently with electro-encephalography (EEG)] (Tong et al., 2005), motor stimulation [concurrently with functional magnetic resonance imaging (fMRI)] (Sassaroli et al., 2006), different levels of mental workload (Sassaroli et al., 2008), cognitive multitasking (Solovey et al., 2011), different sleep stages (Pierro et al., 2012), a cycling exercise (Lin et al., 2013a), arterial blood pressure oscillations (Tgavalekos et al., 2016) and transients (Kainerstorfer et al., 2015) induced by pneumatic cuffs on the subject's legs, electrical stimulation concurrent with voluntary movement (Lin T.-Y. et al., 2016), and fluctuations in intracranial pressure (Ruesch et al., 2019). One may wonder why the phase data was not considered in these studies. In some cases, it is possible that the most common objective of using phase data in FD-NIRS, namely their combination with intensity data to generate absolute measurements of optical properties, was not central to the study. In other cases, there may be reasons such as the lower signal-to-noise ratio of phase data, or uncertainties in how to exploit the added information content of phase data.

Some studies of brain functional activation have compared results obtained with stand-alone DC with those obtained with stand-alone phase (Sassaroli et al., 2004) or with combined DC and phase (Doulgerakis et al., 2019) to explore the importance of the additional information content of phase data. These studies concluded that phase data feature greater sensitivity to deeper, i.e., cerebral, tissue compared to DC data. This point is further explored in Section "Enhanced Depth Sensitivity Using Phase Data" on the depth sensitivity of stand-alone phase measurements.

\section{Stand-Alone Use of AC Data}

While the information content of AC data is essentially the same as for DC data, at least at modulation frequencies typically used in FD-NIRS (say, $<150 \mathrm{MHz}$ ), the AC amplitude is weakly affected by room light or stray light, which instead may significantly 

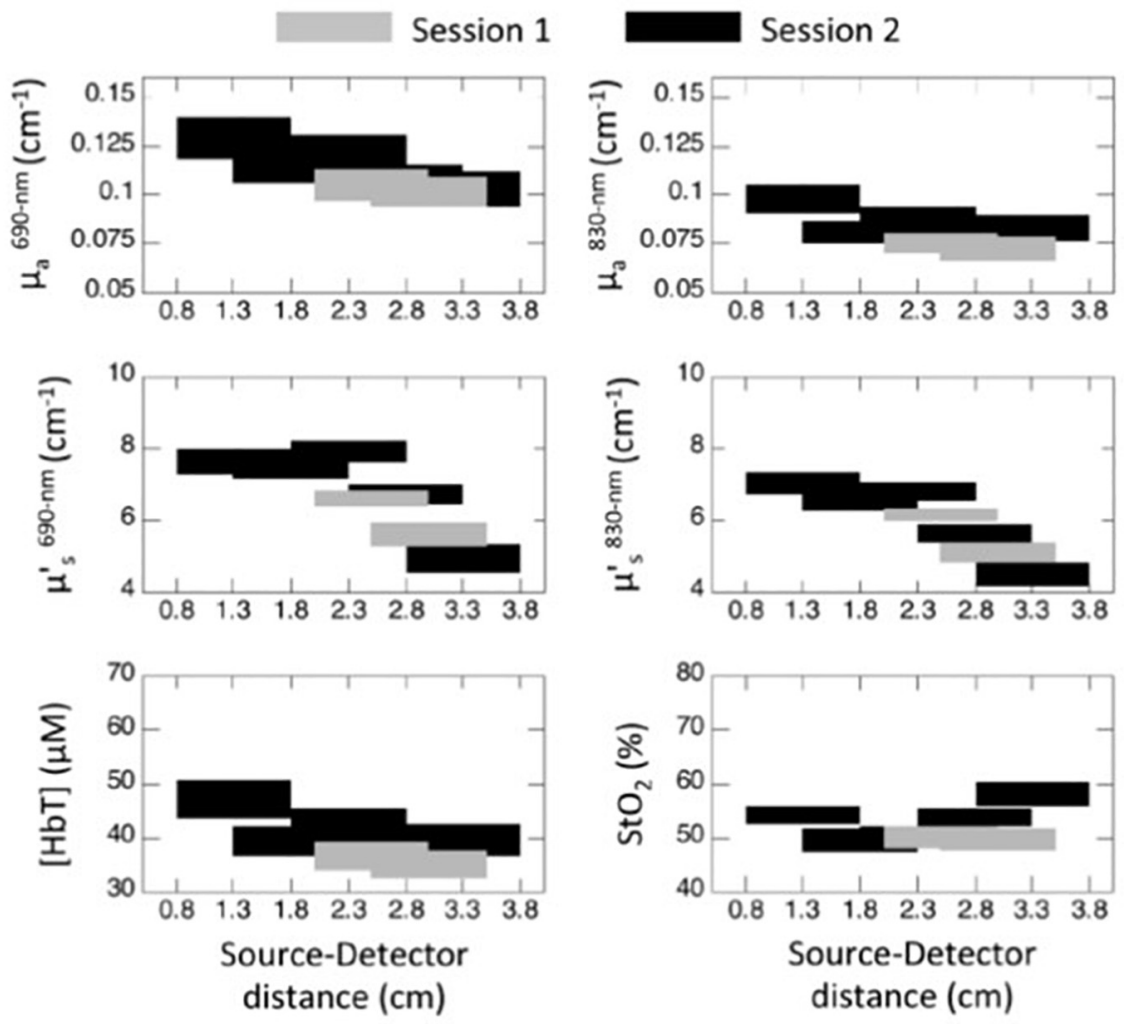

FIGURE 2 | Absolute absorption coefficients ( $\mu_{a}$ : top panels) and reduced scattering coefficients ( $\mu_{s}^{\prime}$ : middle panels) measured with multi-distance FD-NIRS at $690 \mathrm{~nm}$ (left panels) and $830 \mathrm{~nm}$ (right panels) on the forehead of 16 elderly subjects ( $85 \pm 6$ years old) using diffusion theory for a homogeneous semi-infinite medium. From the absorption coefficients at two wavelengths, absolute values of concentration of hemoglobin $([\mathrm{HbT}])$ and tissue saturation $\left(\mathrm{StO}_{2}\right)$ were obtained (bottom panels). The blocks represent the mean \pm standard error (vertical dimension) of the measurements performed at the range of distances corresponding to the horizontal range. The black and gray blocks correspond to two measurement sessions performed on the same group of 16 subjects 5 months apart. Reprinted with permission from Hallacoglu et al. (2012).

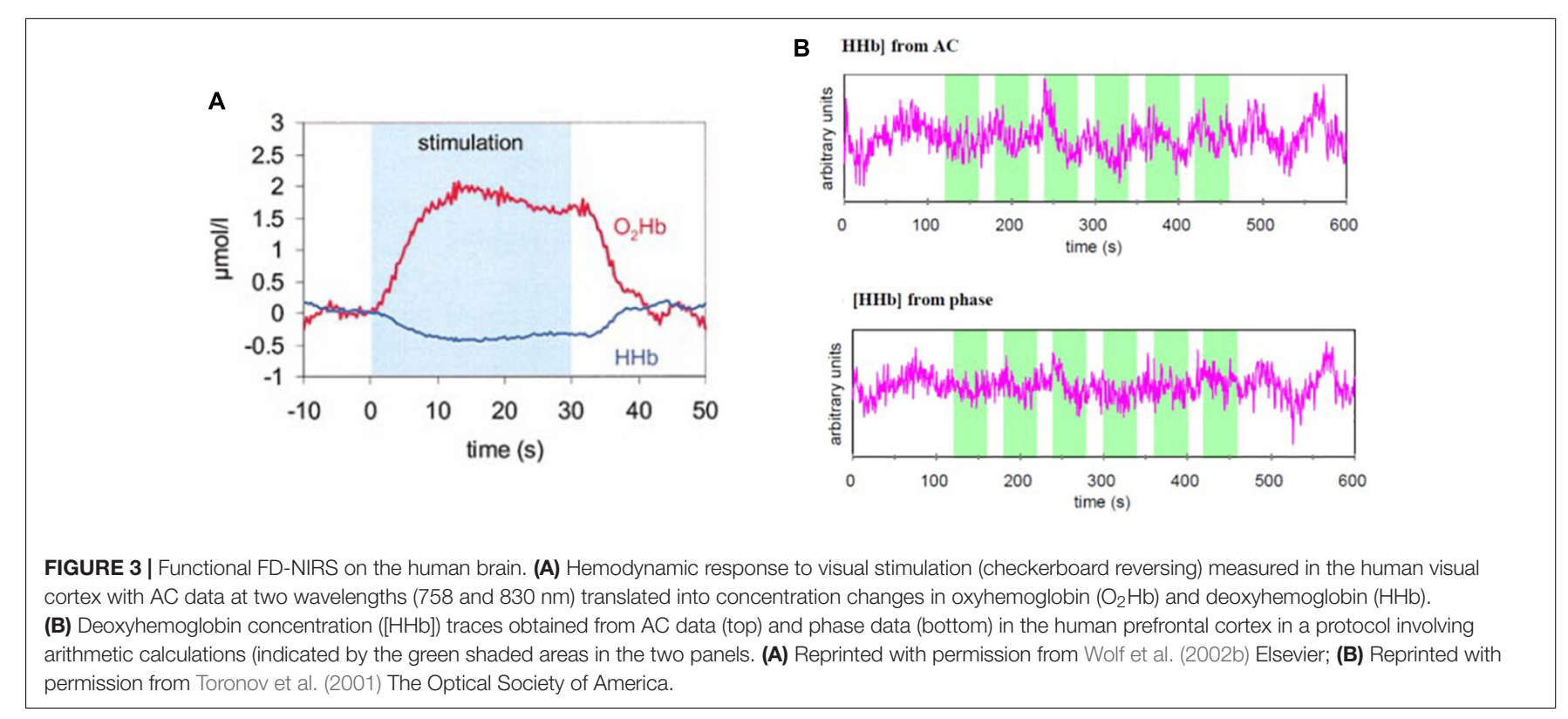


impact the DC intensity. For this reason, some researchers opted to use frequency-domain instrumentation to collect AC data that is then analyzed using data processing techniques typically associated with CW-NIRS (modified Beer-Lambert law with assumed values of DPF). This approach has been used for braincomputer interface research in the areas of decision making (Luu and Chau, 2009), classification of prefrontal activity (Power et al., 2010), for the assessment of independent component analysis (ICA) and principal component analysis (PCA) in removing extracerebral tissue contributions to the NIRS signal (Virtanen et al., 2009), and to investigate the hemodynamics in the prefrontal cortex during arithmetic calculations (Toronov et al., 2001), in the frontal cortex during breath holding, hyperventilation, and sleep (Noponen et al., 2003), in the motor and visual cortices during functional activation (Wolf et al., 2002a,b), and in the visual cortex under normo-, hypo-, and hypercapnia conditions (Noponen et al., 2005). Figure 3A shows the hemodynamic response to visual stimulation by checkerboard reversing in the human visual cortex measured with $\mathrm{AC}$ data, showing the typical increase in oxyhemoglobin and decrease in deoxyhemoglobin associated with the activation-induced increase in cerebral blood flow (Wolf et al., 2002b). The top panel of Figure 3B shows the decrease in deoxyhemoglobin concentration, measured with AC data, in the human prefrontal cortex as a result of arithmetic calculations (Toronov et al., 2001). Note the latency of several seconds for the hemodynamic response to brain activation.

While CW-NIRS data are conceptually equivalent to DC data in FD-NIRS, it is worth noting that it is common for CW-NIRS instruments to use frequency-encoding approaches to distinguish data from different light sources. While the modulation frequency in these cases is on a scale of $\mathrm{kHz}$, thus inappropriate for FD-NIRS, it nevertheless results in a suppression of room light contributions, similarly to AC data in FD-NIRS.

\section{Stand-Alone Use of Phase Data}

While the signal-to-noise ratio associated with phase data in FD-NIRS is typically lower than that of DC and AC data, the unique information content of phase data and the different region of sensitivity of phase vs. DC or AC data (see Figure 1) justify a careful consideration of stand-alone phase. Regarding signal-to-noise ratio, it has been argued that higher modulation frequencies in the range $400-500 \mathrm{MHz}$ may result in a significant improvement over phase measurements at the typical modulation frequencies of $\sim 100 \mathrm{MHz}$ used in FD-NIRS (Toronov et al., 2004).

Stand-alone phase data have been used for quantitative brain oximetry (i.e., measurements of $\mathrm{StO}_{2}$ ), after assuming values for the reduced scattering coefficient of tissue (Sevick et al., 1991; Wilson et al., 1992), or after calibration on known phantoms (Kurth and Thayer, 1999). These methods were applied to newborn piglets during a hypoxic challenge (Ntziachristos et al., 1997), and to human newborns and children with congenital heart disease (Watzman et al., 2000) (see Tables 1, 2).

In the case of fNIRS, one is typically interested in hemodynamic changes associated with brain activation. Such hemodynamic changes affect both intensity and phase data and are mostly associated with absorption changes due to blood volume and blood oxygenation dynamics induced by brain activity. Under the assumption of negligible scattering changes, temporal phase data can be translated into measurements of $\Delta[\mathrm{Hb}]$ and $\Delta\left[\mathrm{HbO}_{2}\right]$ (concentration changes with respect to baseline) (Toronov et al., 2001; Blaney et al., 2020) [see Eq. (6)], similar to the way intensity changes are translated into $\Delta[\mathrm{Hb}]$ and $\Delta\left[\mathrm{HbO}_{2}\right]$ by the modified Beer-Lambert law in CW NIRS (Delpy et al., 1988; Sassaroli and Fantini, 2004) [see Eq. (5)]. Phase-only measurements on the human prefrontal cortex during a protocol involving arithmetic calculations (Toronov et al., 2001) and on the primary motor cortex during a finger-tapping task (Sassaroli et al., 2004) demonstrated the ability of stand-alone phase data to detect brain activation. The bottom panel of Figure $\mathbf{3 B}$ shows the decrease in deoxyhemoglobin concentration, measured with phase data, in the human prefrontal cortex as a result of arithmetic calculations (Toronov et al., 2001).

\section{The Fast Optical Signal}

FD-NIRS has been applied to the study of the so-called fast optical signal (occurring on a time scale of $\sim 100 \mathrm{~ms}$ ), which is thought to be associated with scattering changes that are more directly representative of neuronal activation than the absorption changes associated with cerebral hemodynamic responses (occurring on a time scale of seconds). The fast optical signal was first reported with phase measurements in the human visual cortex under the name EROS, for "event-related optical signal" (Gratton et al., 1995a). Phase measurements, and sometimes AC measurements, were then used to investigate the fast optical signal in the visual (Gratton and Fabiani, 2003; Gratton et al., 2006; Maclin et al., 2007), auditory (Maclin et al., 2003), somatosensory (Maclin et al., 2004), motor (Gratton et al., 1995b; Parks et al., 2012), temporal/frontal (Tse et al., 2006, 2013; Tse and Penney, 2007), and prefrontal (Low et al., 2006, 2009; Gratton et al., 2009; Huang et al., 2013; Baniqued et al., 2013) cortices. Brain activation was reported to evoke a fast, transient phase increase of the order of $0.05^{\circ}$ (sometimes extending to values of $0.1^{\circ}$ or $0.2^{\circ}$ ) (see Figure 4A).

Stand-alone DC data, AC data, and phase data have been considered in the investigation of the fast optical signal. Some human studies on the motor cortex (Wolf et al., 2002a; Morren et al., 2004), visual cortex (Wolf et al., 2003), and prefrontal cortex (Proulx et al., 2018a,b) reported that DC and AC data can detect the fast signal more effectively than phase data. The fast optical signal was also investigated with CW-NIRS in the somatosensory cortex (Steinbrink et al., 2000), prefrontal cortex (Medvedev et al., 2010), temporal cortex (Kubota et al., 2008), visual cortex (Sun et al., 2014), and motor cortex (Zhang et al., 2012). However, given the small amplitude of the fast optical signal, questions were raised about its detectability (Steinbrink et al., 2005). In fact, the fast optical signal consists of a small transient decrease in the DC intensity (or AC amplitude), of the order of $0.05 \%$ or less (see Figure 4B). (We note that an animal study on the somatosensory cortex of marmosets found a transient increase of $\sim 0.08 \%$ in the CW intensity, in a method termed optoencephalography; 

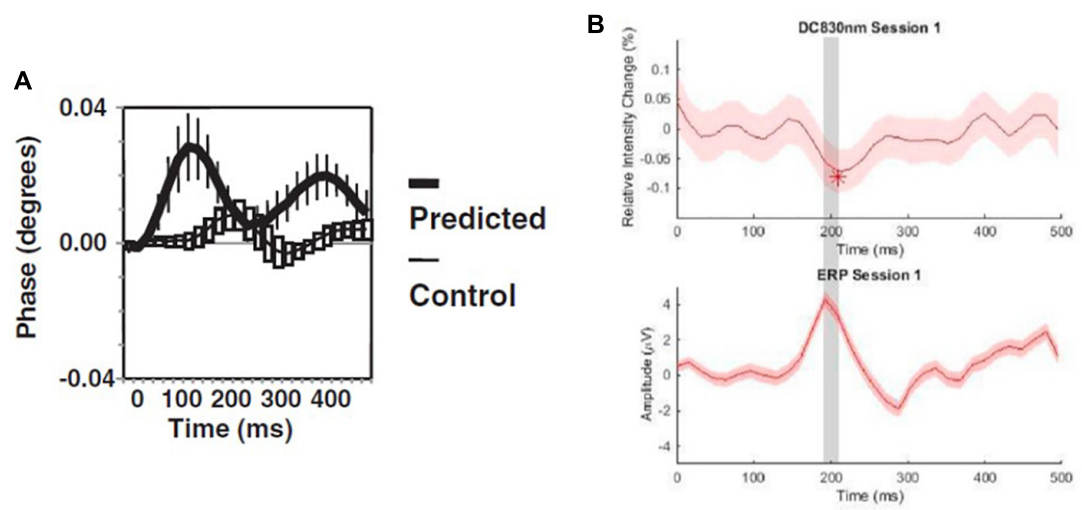

FIGURE 4 | Fast optical signal measured by FD-NIRS with (A) phase from the visual cortex of human subjects at the cortical location of predicted response and at a control location (EROS: event-related optical signal), and (B) DC Intensity from the prefrontal cortex (top) in comparison with the event-related potential (ERP) response. (A) Reprinted with permission from Gratton and Fabiani (2003)@ Wiley; (B) Reprinted with permission from Proulx et al. (2018b)(C) Elsevier.

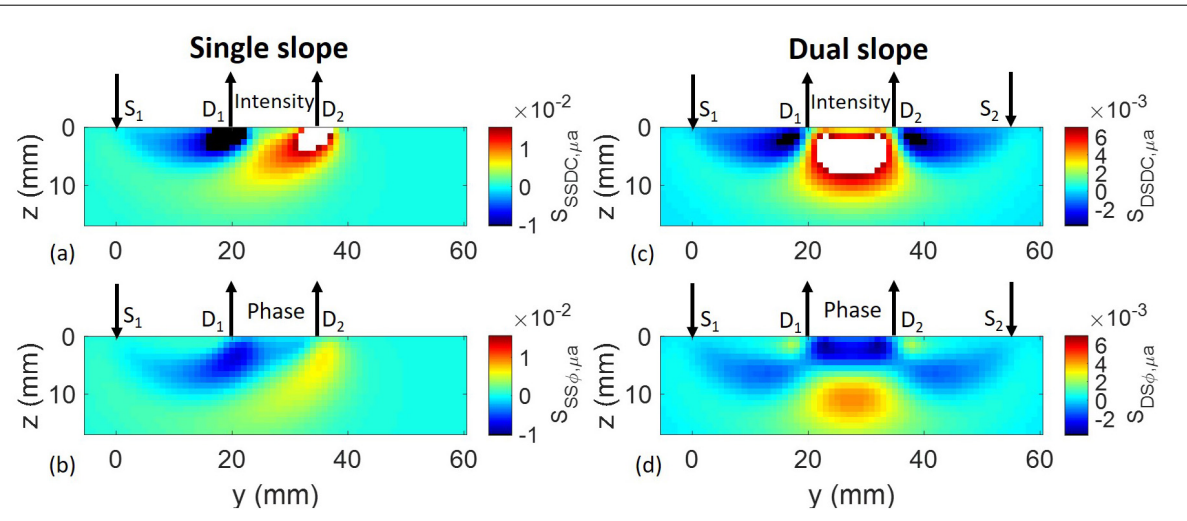

FIGURE 5 | Sensitivity maps for absorption contrast of (A) single-slope intensity, (B) single-slope phase, (C) dual-slope intensity, and (D) dual-slope phase. The arrows pointing down indicate the light sources $\left(S_{1}, S_{2}\right)$, and the arrows pointing up indicate the optical detectors $\left(D_{1}, D_{2}\right)$. The color bar labels in panels $(\mathbf{A}, \mathbf{C})$ indicate the sensitivity to absorption perturbations for single slope DC intensity $\left(S_{S S D C, \mu_{a}}\right)$ and dual slope DC intensity $\left(S_{D S D C, \mu_{a}}\right)$, respectively. The color bar labels in panels (B,D) indicate the sensitivity to absorption perturbations for single slope phase $\left(S_{S} S_{\varphi, \mu_{a}}\right)$ and dual slope phase $\left(S_{D S} \varphi, \mu_{a}\right)$, respectively. White and black in the color maps indicate values greater than the maximum or smaller than the minimum, respectively, of the color bars. Adapted with permission from Sassaroli et al. (2019)(C) The Optical Society of America.

Hiwaki and Miyaguchi, 2018). The challenge of detecting the fast optical signal was further demonstrated in studies of the human motor cortex (Franceschini and Boas, 2004) and the primate visual cortex (Radhakrishnan et al., 2009).

\section{Enhanced Depth Sensitivity Using Phase Data}

A comparison of Figures 1A,B shows that single-distance phase measurements feature a sensitivity to absorption perturbations that extends deeper than single-distance intensity measurements. In fact, as already mentioned, it was observed that phase measurements provide deeper sensitivity, and thus a stronger sensitivity to cerebral tissue in non-invasive optical studies of the human brain (Sassaroli et al., 2004; Doulgerakis et al., 2019). In an effort to minimize the sensitivity to superficial tissue layers, multi-distance measurements were introduced in both CW and FD-NIRS, and implemented with either a single source and multiple detectors, or a single detector and multiple sources (Franceschini et al., 1998; Suzuki et al., 1999). Since these methods ultimately aimed at measuring a gradient, or slope, of optical signals versus source-detector distance, we refer to these methods as single-slope methods. These methods have been extensively used in both CW-NIRS and FD-NIRS, and have been implemented into several commercial NIRS instruments. While single-slope methods indeed feature a small sensitivity to uniform absorption changes in superficial layers, they may yield misleading results in the case of localized superficial perturbations. In fact, as shown in Figures $\mathbf{5 A}, \mathbf{B}$, the single-slope sensitivity of both intensity and phase feature double, asymmetrical banana-shaped regions with sensitivities of opposite sign (Sassaroli et al., 2019). This feature may be problematic in the case of localized superficial inhomogeneous changes in optical properties.

We have recently proposed to enhance depth sensitivity using a particular source-detector arrangement that was originally 
introduced for self-calibrating, absolute measurements of optical properties with FD-NIRS (Hueber et al., 1999) (as described in Section "Multi-Distance Methods"). This particular arrangement involves at least two sources and at least two detectors to measure two paired gradients or slopes of optical signals versus sourcedetector distance, which are then averaged. Because it is based on the measurement of two slopes, this approach is referred to as a dual-slope method, and it can be applied separately to intensity and phase data. The region of sensitivity of dual slopes for intensity and phase, in the case of a linear arrangement of two sources and two detectors, is illustrated in Figures 5C,D for the same optical properties and modulation frequency of Figure 1. In this case, however, we considered a larger absorption perturbation having an axial size (along $z$ ) of $1 \mathrm{~mm}$ and a lateral size (across $x$ and $y$ ) of $5 \mathrm{~mm}$, which may represent a typical size of localized hemodynamic changes (as related to functional brain activation or extracerebral hemodynamics). A variety of other source-detector arrangements for dual-slope measurements, and the corresponding intensity and phase regions of sensitivity, have also been reported (Fantini et al., 2020). The dual-slope sensitivity features both positive and negative values, and takes maximum values deeper in the tissue than the single-distance sensitivity (in the case of Figure 5: $\sim 5 \mathrm{~mm}$ vs. $\sim 2 \mathrm{~mm}$ for intensity, and $\sim 11 \mathrm{~mm}$ vs. $\sim 5 \mathrm{~mm}$ for phase). Furthermore, it is evident from Figures 5 C,D that the sensitivity of phase dual-slope extends deeper than the sensitivity of intensity dualslope. An FD-NIRS study on human subjects showed the different information content of intensity and phase collected with singledistance, single-slope, and dual-slope arrangements, consistent with a deeper sensitivity of phase slope methods, and more robust dual-slope vs. single-slope data (Blaney et al., 2020).

The above observations about single-slope and dual-slope sensitivities have also been articulated in the time domain, where source-detector arrangements for self-calibrating absolute measurements have been shown to achieve a better depth sensitivity with the first and second moments of the photon timeof-flight distribution (the first moment is representative of the phase in the frequency domain), with respect to the 0th moment (which represents the CW intensity) (Sawosz and Liebert, 2019).

\section{CRITICAL PERSPECTIVE: WHAT IS THE MAJOR PROMISE OF FD DATA IN CEREBRAL NIRS?}

FD-NIRS provides data with richer information content than CW-NIRS. In this article, we have discussed how such additional information may be used in three broad areas: (1) absolute quantification of baseline optical properties and hemoglobin

\section{REFERENCES}

Applegate, M. B., and Roblyer, D. (2018). Multi-distance diffuse optical spectroscopy with a single optode via hypotrochoidal scanning. Opt. Lett. 43, 747-750. doi: 10.1364/OL.43.000747

Arnesano, C., Santoro, Y., and Gratton, E. (2012). Digital parallel frequencydomain spectroscopy for tissue imaging. J. Biomed. Opt. 17:096014. concentration in tissue; (2) minimization of sensitivity to room light and other potential sources of confounds (such as instrumental drifts, variable optical coupling with tissue, and motion artifacts) in practical measurements; (3) achievement of selective sensitivity to deeper tissue.

The ability to perform absolute baseline measurements is undoubtedly appealing, since it leads to the possibility of determining normative baseline values, performing comparisons across subjects, and conducting longitudinal studies over long periods of time. However, the accuracy of absolute measurements may be significantly affected by simplifying assumptions (on the tissue geometry, spatial distribution of optical properties, etc.), and by instrumental or experimental artifacts that are often difficult to avoid in real-life measurement conditions. These assumptions and experimental artifacts can be particularly limiting in the case of non-invasive brain studies because of the strong tissue heterogeneity, presence of hair, tissue rigidity due to the skull bone, etc. Furthermore, many applications of functional NIRS or cerebral oximetry do not need an absolute baseline characterization, but rather rely on relative changes during specific measurement protocols.

Practical advantages related to a lack of sensitivity to room light or other sources of instrumental artifacts are important, but may also be achieved with alternative methods.

In our opinion, the one aspect of FD-NIRS that holds the most promise to advance the field of non-invasive optical sensing of the brain is the ability to control the region of sensitivity by taking advantage of intensity and phase measurements collected with properly designed source-detector arrays. Such a capability is not available to CW-NIRS, and can help address one of the most critical open questions in the field, namely the minimization of confounding contributions from extracerebral tissue (scalp, skull, etc.) to non-invasive optical measurements of the brain. The possibility of achieving more selective sensitivity to brain tissue, while also providing access to valuable absolute measurements of optical properties and chromophore concentrations, renders FD-NIRS a powerful technique for non-invasive measurements of the human brain.

\section{AUTHOR CONTRIBUTIONS}

SF and AS: literature survey, analysis, and writing. SF: tables.

\section{FUNDING}

We acknowledge support from the US National Institutes of Health (NIH), Grant No. R01 NS095334.

Baniqued, P. L., Low, K. A., Fabiani, M., and Gratton, G. (2013). Frontoparietal traffic signals; a fast optical imaging study of preparatory dynamics in response mode switching. J. Cogn. Neurosci. 25, 887-902. doi: 10.1162/jocn_a_ 00341

Bevilacqua, F., Berger, A. J., Cerussi, A. E., Jakubowski, D., and Tromberg, B. J. (2000). Broadband absorption spectroscopy in turbid media by combined frequency-domain and steady-state methods. Appl. Opt. 39, 6498-6507. 
Bigio, I. J., and Fantini, S. (2016). Quantitative Biomedical Optics. Cambridge: Cambridge University Press.

Blaney, G., Sassaroli, A., Pham, T., Fernandez, C., and Fantini, S. (2020). Phase dual slopes in frequency-domain near-infrared spectroscopy for enhanced sensitivity to brain tissue: first applications to human subjects. J. Biophotonics 13:e201960018. doi: 10.1002/jbio.201960018

Blaney, G., Sassaroli, A., Pham, T., Krishnamurthy, N., and Fantini, S. (2019). Multi-distance frequency-domain optical measurements of coherent cerebral hemodynamics. Photonics 6:83.

Boas, D. A. (1997). A fundamental limitation of linearized algorithms for diffuse optical tomography. Opt. Express 13, 404-413.

Calderon-Arnulphi, M., Alaraj, A., Amin-Hanjani, S., Mantulin, W. W., Polzonetti, C. M., Gratton, E., et al. (2007). Detection of cerebral ischemia in neurovascular surgery using quantitative frequency-domain near-infrared spectroscopy. J. Neurosurg. 106, 283-290.

Cerussi, A. E., and Tromberg, B. J. (2003). "Photon migration spectroscopy frequency-domain techniques," in Biomedical Photonics Handbook, ed. T. V. Dinh (Boca Raton, FL: CRC Press), 22/1-22/17.

Chance, B., Anday, E., Nioka, S., Zhou, S., Hong, L., Worden, K., et al. (1998a). A novel method for fast imaging of brain function, non-invasively, with light. Opt. Express 2, 411-423.

Chance, B., Cope, M., Gratton, E., Ramanujam, N., and Tromberg, B. (1998b). Phase measurement of light absorption and scatter in human tissue. Rec. Sci. Instrum. 69, 3457-3481.

Chance, B., Kang, K., He, L., Weng, J., and Sevick, E. (1993). Highly sensitive object location in tissue models with linear in-phase and anti-phase multi-element optical arrays in one and two dimensions. Proc. Natl. Acad. Sci. U.S.A. 90, 3423-3427.

Chance, B., Kang, K. A., He, L., Liu, H., and Zhou, S. (1996). Precision localization of hidden absorbers in body tissues with phased-array optical systems. Rev. Sci. Instrum. 67, 4324-4332.

Cheung, C., Culver, J. P., Takahashi, K., Greenberg, J. H., and Yodh, A. G. (2001). In vivo cerebrovascular measurement combining diffuse near-infrared absorption and correlation spectroscopies. Phys. Med. Biol. 46, 2053-2065.

Clancy, M., Belli, A., Davies, D., Lucas, S. J. E., Su, Z., and Dehghani, H. (2015). Comparison of neurological NIRS signals during standing Valsalva maneuvers, pre and post vasopressor injection. Proc. SPIE 9538:953817.

Culver, J. P., Durduran, T., Furuya, D., Cheung, C., Greenberg, J. H., and Yodh, A. G. (2003). Diffuse optical tomography of cerebral blood flow, oxygenation, and metabolism in rat during focal ischemia. J. Cereb. Blood Flow Metab. 23, 911-924.

Davies, D. J., Clancy, M., Dehghani, H., Lucas, S. J. E., Forcione, M., Yakoub, K. M., et al. (2019). Cerebral oxygenation in traumatic brain injury: can a noninvasive frequency domain near-infrared spectroscopy device detect changes in brain tissue oxygen tension as well as the established invasive monitor? J. Neurotrauma 36, 1175-1183. doi: 10.1089/neu.2018.5667

Davies, D. J., Clancy, M., Lighter, D., Balanos, G. M., Lucas, S. J. E., Dehghani, H., et al. (2017). Frequency-domain vs continuous-wave nearinfrared spectroscopy devices: a comparison of clinically viable monitors in controlled hypoxia. J. Clin. Monit. Comput. 31, 967-974. doi: 10.1007/s10877016-9942-5

Dehaes, M., Aggarwal, A., Lin, P. Y., Fortuno, C. R., Fenoglio, A., Roche-Labarbe, N., et al. (2014). Cerebral oxygen metabolism in neonatal hypoxic ischemic encephalopathy during and after therapeutic hypothermia. J. Cereb. Blood Flow Metab. 34, 87-94. doi: 10.1038/jcbfm.2013.165

Dehaes, M., Cheng, H. H., Buckley, E. M., Lin, P.-Y., Ferradal, S. L., Williams, K., et al. (2015). Perioperative cerebral hemodynamics and oxygen metabolism in neonates with single-ventricle physiology. Biomed. Opt. Express 6, 4749-4767. doi: 10.1364/BOE.6.004749

Delpy, D. T., Cope, M., van der Zee, P., Arridge, S., Wray, S., and Wyatt, J. (1988). Estimation of optical path length measurements on adult head, calf, and forearm and the head of newborn infants using phase resolved spectroscopy. Phys. Med. Biol. 40, 295-304.

Demel, A., Feilke, K., Schöning, M., Wolf, M., Poets, C. F., and Franz, A. R. (2015). Healthy term and moderately preterm infants have similar cerebral oxygen saturation and cerebral flow volumes during early post-natal transition. Acta Paediatr. 104, e330-e336. doi: 10.1111/apa.13023
Demel, A., Feilke, K., Wolf, M., Poets, C. F., and Franz, A. R. (2014a). Correlation between skin, bone, and cerebrospinal fluid layer thickness and optical coefficients measured by multidistance frequency-domain near-infrared spectroscopy in term and preterm infants. J. Biomed. Opt. 19:017004. doi: 10. 1117/1.JBO.19.1.017004

Demel, A., Wolf, M., Poets, C. F., and Franz, A. R. (2014b). Effect of different assumptions for brain water content on absolute measures of cerebral oxygenation determined by frequency-domain near-infrared spectroscopy in preterm infants: an observational study. BMC Pediatr. 14:206. doi: 10.1186/ 1471-2431-14-206

Doulgerakis, M., Eggebrecht, A. T., and Dehghani, H. (2019). High-density functional diffuse optical tomography based on frequency-domain measurements improves image quality and spatial resolution. Neurophotonics 6:035007. doi: 10.1117/1.NPh.6.3.035007

Fabbri, F., Henry, M. E., Renshaw, P. F., Nadgir, S., Ehrenberg, B. L., Franceschini, M. A., et al. (2003). Bilateral near-infrared monitoring of the cerebral concentration and oxygen-saturation of hemoglobin during right unilateral electro-convulsive therapy. Brain Res. 992, 193-204.

Fantini, S., Aggarwal, P., Chen, K., Franceschini, M. A., and Ehrenberg, B. L. (2003). Near-infrared spectroscopy and polysomnography during all-night sleep in human subjects. Proc. SPIE 5068, 155-162.

Fantini, S., Barbieri, B., Franceschini, M. A., and Gratton, E. (1997). "Frequencydomain spectroscopy," in Applications of Optical Engineering to the Study of Cellular Pathology, ed. E. Kohen (Thiruvananthapuram: Research Signpost), $57-66$.

Fantini, S., Blaney, G., and Sassaroli, A. (2020). Transformational change in the field of diffuse optics: from going bananas to going nuts. J. Innov. Opt. Health Sci. 13:1930013.

Fantini, S., Franceschini, M. A., Fishkin, J. B., Barbieri, B., and Gratton, E. (1994a). Quantitative determination of the absorption spectra of chromophores in strongly scattering media: a light-emitting-diode based technique. Appl. Opt. 33, 5204-5213. doi: 10.1364/AO.33.005204

Fantini, S., Franceschini, M. A., and Gratton, E. (1994b). Semi-infinite-geometry boundary problem for light migration in highly scattering media: a frequencydomain study in the diffusion approximation. J. Opt. Soc. Am. B 10, 2128-2138.

Fantini, S., Franceschini-Fantini, M. A., Maier, J. S., Walker, S. A., Barbieri, B., and Gratton, E. (1995). Frequency-domain multichannel optical detector for noninvasive tissue spectroscopy and oximetry. Opt. Eng. 34, 32-42.

Fantini, S., Frederick, B., and Sassaroli, A. (2018). Perspective: prospects of noninvasive sensing of the human brain with diffuse optical imaging. APL Photonics 3:110901. doi: 10.1063/1.5038571

Fantini, S., Hueber, D., Franceschini, M. A., Gratton, E., Rosenfeld, W., Stubblefield, P. G., et al. (1999). Non-invasive optical monitoring of the newborn piglet brain using continuous-wave and frequency-domain spectroscopy. Phys. Med. Biol. 44, 1543-1563.

Fantini, S., and Sassaroli, A. (2016). "Frequency-domain techniques for tissue spectroscopy and imaging," in Handbook of Optical Biomedical Diagnostics: Light-Tissue Interactions, 2nd Edn, Vol. 1, ed. V. V. Tuchin (Bellingham, WA: SPIE Press), 477-532.

Farzam, P., Buckley, E. M., Lin, P. Y., Hagan, K., Grant, P. E., Inder, T. E., et al. (2017). Shedding light on the neonatal brain: probing cerebral hemodynamics by diffuse optical spectroscopic methods. Sci. Rep. 7:15786.

Feng, S., Zeng, F., and Chance, B. (1995). Photon migration in the presence of a single defect: a perturbation analysis. Appl. Opt. 34, 3826-3837. doi: 10.1364/ AO. 34.003826

Ferradal, S. L., Yuki, K., Vyas, R., Ha, C. G., Yi, F., Stopp, C., et al. (2017). Non-invasive assessment of cerebral blood flow and oxygen metabolism in neonates during hypothermic cardiopulmonary bypass: feasibility and clinical implications. Sci. Rep. 7:44117. doi: 10.1038/srep44117

Ferrari, M., and Quaresima, V. (2012). A brief review on the history of human functional near-infrared spectroscopy (fNIRS) development and fields of application. Neuroimage 63, 921-935. doi: 10.1016/j.neuroimage.2012.03.049

Fishkin, J. B., and Gratton, E. (1993). Propagation of photon-density waves in strongly scattering media containing an absorbing semi-infinite plane bounded by a straight edge. J. Opt. Soc. Am. A 10, 127-140.

Franceschini, M. A., and Boas, D. A. (2004). Noninvasive measurement of neuronal activity with near-infrared optical imaging. Neuroimage 21, 372-386. 
Franceschini, M. A., Fantini, S., Paunescu, L. A., Maier, J. S., and Gratton, E. (1998). Influence of a superficial layer in the quantitative spectroscopic study of strongly scattering media. Appl. Opt. 37, 7447-7458.

Franceschini, M. A., Thaker, S., Themelis, G., Krishnamoorthy, K. K., Bortfeld, H., Diamond, S. G., et al. (2007). Assessment of infant brain development with frequency-domain near-infrared spectroscopy. Pediatr. Res. 61, 546-551.

Gatto, R., Hoffman, W., Mueller, M., Flores, A., Valyi-Nagy, T., and Charbel, F. T. (2006). Frequency domain near-infrared spectroscopy technique in the assessment of brain oxygenation: a validation study in live subjects and cadaver. J. Neurosci. Methods 157, 274-277.

Gatto, R., Hoffman, W., Paisansathan, C., Mantulin, W., Gratton, E., and Charbel, F. T. (2007). Effect of age on brain oxygenation regulation during changes in position. J. Neurosci. Methods 164, 308-311.

Grant, P. E., Roche-Labarbe, N., Surova, A., Themelis, G., Selb, J., Warren, E. K., et al. (2009). Increased cerebral blood volume and oxygen consumption in neonatal brain injury. J. Cereb. Blood Flow Metab. 29, 1704-1713. doi: 10.1038/ jcbfm. 2009.90

Gratton, G., Brumback, C. R., Gordon, B. A., Pearson, M. A., Low, K. A., and Fabiani, M. (2006). Effects of measurements method, wavelength, and sourcedetector distance on the fast optical signal. Neuroimage 32, 1576-1590.

Gratton, G., Corballis, P. M., Cho, E., Fabiani, M., and Hood, D. C. (1995a). Shades of gray matter: noninvasive optical images of human brain responses during visual stimulation. Psychophysiology 32, 505-509.

Gratton, G., Fabiani, M., Friedman, D., Franceschini, M. A., Fantini, S., Corballis, P., et al. (1995b). Rapid changes of optical parameters in the human brain during a tapping task. J. Cogn. Neurosci. 7, 446-456. doi: 10.1162/jocn.1995. 7.4 .446

Gratton, G., and Fabiani, M. (2003). The event-related optical signal (EROS) in visual cortex: replicability, consistency, localization, and resolution. Psychophysiology 40, 561-571.

Gratton, G., Wee, E., Rykhlevskaia, E. I., Leaver, E. E., and Fabiani, M. (2009). Does white matter matter? Spatio-temporal dynamics of task switching in aging. J. Cogn. Neurosci. 21, 1380-1395. doi: 10.1162/jocn.2009.21093

Gulsen, G., Xiong, B., Birgul, O., and Nalcioglu, O. (2006). Design and implementation of a multifrequency near-infrared diffuse optical tomography system. J. Biomed. Opt. 11:014020.

Hallacoglu, B., Sassaroli, A., and Fantini, S. (2013). Optical characterization of two-layered turbid media for non-invasive, absolute oximetry in cerebral and extracerebral tissue. PLoS One 8:e64095. doi: 10.1371/journal.pone.006 4095

Hallacoglu, B., Sassaroli, A., Wysocki, M., Guerrero-Berroa, E., Schnaider Beeri, M., Haroutunian, V., et al. (2012). Absolute measurement of cerebral optical coefficients, hemoglobin concentration and oxygen saturation in old and young adults with near-infrared spectroscopy. J. Biomed. Opt. 17:081406. doi: 10.1117/ 1.JBO.17.8.081406

Haskell, R. C., Svaasand, L. O., Tsay, T., Feng, T., McAdams, M. S., and Tromberg, B. J. (1994). Boundary conditions for the diffusion equation in radiative transfer. J. Opt. Soc. Am. A 10, 2727-2741.

Hiwaki, O., and Miyaguchi, H. (2018). Noninvasive measurement of dynamic brain signals using light penetrating the brain. PLoS One 13:e0192095. doi: 10.1371/journal.pone.0192095

Huang, J., Wang, S., Jia, A., Mo, D., and Chen, H.-C. (2013). Cortical dynamics of semantic processing during sentence comprehension: evidence from eventrelated optical signals. PLoS One 8:e70671. doi: 10.1371/journal.pone.0070671

Hueber, D. M., Fantini, S., Cerussi, A. E., and Barbieri, B. B. (1999). New optical design for absolute (self-calibrating) NIR tissue hemoglobin measurements. Proc. SPIE 3597, 618-631.

Intes, X., Ntziachristos, V., and Chance, B. (2002). Analytical model for dualinterfering sources diffuse optical tomography. Opt. Express 10, 2-14.

Irani, F., Platek, S. M., Bunce, S., Ruocco, A. C., and Chute, D. (2007). Functional near infrared spectroscopy (fNIRS): an emerging neuroimaging technology with important applications for the study of brain disorders. Clin. Neuropsychol. $21,9-37$.

Kainerstorfer, J. M., Sassaroli, A., Tgavalekos, K. T., and Fantini, S. (2015). Cerebral autoregulation in the microvasculature measured with near-infrared spectroscopy. J. Cereb. Blood Flow Metab. 35, 955-966.

Kitsmiller, V. J., Dummer, M. M., Johnson, K., Cole, G. D., and Sullivan, T. D. O. (2018). Frequency domain diffuse optical spectroscopy with a near-infrared tunable vertical cavity surface emitting laser. Opt. Express 26, 21033-21043. doi: 10.1364/OE.26.021033

Kubota, M., Inouchi, M., Dan, I., Tsuzuki, D., Ishikawa, A., and Scovel, T. (2008). Fast (100-175 ms) components elicited bilaterally by language production as measured by three-wavelength optical imaging. Brain Res. 1226, 124-133. doi: 10.1016/j.brainres.2008.05.079

Kurth, C. D., and Thayer, W. S. (1999). A multiwavelength frequency-domain near-infrared cerebral oximeter. Phys. Med. Biol. 44, 727-740.

la Cour, A., Greisen, G., and Hyttel-Sorensen, S. (2018). In vivo validation of cerebral near-infrared spectroscopy: a review. Neurophotonics 5:040901. doi: 10.1117/1.NPh.5.4.040901

Lin, P.-Y., Hagan, K., Fenoglio, A., Grant, P. E., and Franceschini, M. A. (2016). Reduced cerebral blood flow and oxygen metabolism in extremely preterm neonates with low-grade germinal matrix- intraventricular hemorrhage. Sci. Rep. 6:25903. doi: 10.1038/srep25903

Lin, T.-Y., Wu, J.-S., Lin, L. L., Ho, T.-C., Lin, P.-Y., and Chen, J.-J. (2016). Assessments of muscle oxygenation and cortical activity using functional nearinfrared spectroscopy in healthy adults during hybrid activation. IEEE Trans. Neural Syst. Rehabil. Eng. 24, 1-9.

Lin, P.-Y., Chen, J. J., and Lin, S.-I. (2013a). The cortical control of cycling exercise in stroke patients: an fNIRS study. Hum. Brain Mapp. 34, 2381-2390.

Lin, P.-Y., Roche-Labarbe, N., Dehaes, M., Fenoglio, A., Grant, P. E., and Franceschini, M. A. (2013b). Regional and hemispheric asymmetries of cerebral hemodynamic and oxygen metabolism in newborns. Cereb. Cortex 23, 339-348. doi: 10.1093/cercor/bhs023

Liu, N., Sassaroli, A., and Fantini, S. (2005a). Two-dimensional phased-arrays of sources and detectors for depth discrimination in diffuse optical imaging. J. Biomed. Opt. 10:051801.

Liu, N., Sassaroli, A., Zucker, M. A., and Fantini, S. (2005b). Three-element phased-array approach to diffuse optical imaging based on post-processing of continuous-wave data. Opt. Lett. 30, 281-283.

Low, K. A., Leaver, E., Kramer, A. F., Fabiani, M., and Gratton, G. (2006). Fast optical imaging of frontal cortex during active and passive oddball tasks. Psychophysiology 43, 127-136.

Low, K. A., Leaver, E. E., Kramer, A. F., Fabiani, M., and Gratton, G. (2009). Share or compete? Load-dependent recruitment of prefrontal cortex during dual-task performance. Psychophysiology 46, 1069-1079.

Luu, S., and Chau, T. (2009). Decoding subjective preference from single-trial nearinfrared spectroscopy signals. J. Neural Eng. 6:016003. doi: 10.1088/1741-2560/ 6/1/016003

Maclin, E. L., Gratton, G., and Fabiani, M. (2003). Optimum filtering for EROS measurements. Psychophysiology 40, 542-547.

Maclin, E. L., Low, K. A., Fabiani, M., and Gratton, G. (2007). Improving the signalto-noise ratio of event-related optical signals. IEEE Eng. Med. Biol. Mag. 26, 47-51.

Maclin, E. L., Low, K. A., Sable, J. J., Fabiani, M., and Gratton, G. (2004). The eventrelated optical signal to electrical stimulation of the median nerve. Neuroimage 21, 1798-1804.

Martelli, F., Del Bianco, S., Ismaelli, A., and Zaccanti, G. (2010). Light Propagation through Biological Tissues and Other Diffusive Media. Bellingham, WA: SPIE press.

McBride, T. O., Pogue, B. W., Jiang, S., Österberg, U. L., and Paulsen, K. D. (2001). A parallel-detection frequency-domain near-infrared tomography system for hemoglobin imaging of the breast in vivo. Rev. Sci. Instrum. 72, 1817-1824.

McIntosh, M. A., Shahani, U., Boulton, R. G., and McCulloch, D. L. (2010). Absolute quantification of oxygenated hemoglobin within the visual cortex with functional near infrared spectroscopy (fNIRS). Invest. Ophtalmol. Vis. Sci. 51, 4856-4860. doi: 10.1167/iovs.09-4940

McKernan Ward, L., Aitchison, R. T., Tawse, M., Simmers, A. J., and Shahani, U. (2015). Reduced haemodynamic response in the ageing visual cortex measured by absolute fNIRS. PLoS One 10:e125012. doi: 10.1371/journal.pone.0125012

Medvedev, A. V., Kainerstorfer, J. M., Borisov, S. V., Gandjbakhche, A. H., and VanMeter, J. (2010). 'Seeing' electroencephalogram through the skull: imaging prefrontal cortex with fast optical signal. J. Biomed. Opt. 15:061702.

Meng, L., Gelb, A. W., Alexander, B. S., Cerussi, A. E., Tromberg, B. J., Yu, Z., et al. (2012a). Impact of phenylephrine administration on cerebral tissue oxygen saturation and blood volume is modulated by carbon dioxide in anaesthesized patients. Br. J. Anaesth. 108, 815-822. doi: 10.1093/bja/aes023 
Meng, L., Mantulin, W. W., Alexander, B. S., Cerussi, A. E., Tromberg, B. J., Yu, Z., et al. (2012b). Head-up tilt and hyperventilation produce similar changes in cerebral oxygenation and blood volume: an observational comparison study using frequency-domain near-infrared spectroscopy. Can. J. Anesth. 59, 357365. doi: 10.1007/s12630-011-9662-8

Metz, A. J., Klein, S. D., Scholkmann, F., and Wolf, U. (2017). Continuous coloured light altered human brain haemodynamics, and oxygenation assessed by systemic physiology augmented functional near-infrared spectroscopy. Sci. Rep. 7:10027. doi: 10.1038/s41598-017-09970-z

Moreau, F., Yang, R., Nambiar, V., Demchuk, A. M., and Dunn, J. F. (2016). Near-infrared measurements of brain oxygenation in stroke. Neurophotonics 3:031403. doi: 10.1117/1.NPh.3.3.031403

Morren, G., Wolf, M., Lemmerling, P., Wolf, U., Choi, J. H., Gratton, E., et al. (2004). Detection of fast neuronal signals in the motor cortex from functional near infrared spectroscopy measurements using independent component analysis. Med. Biol. Eng. Comput. 42, 92-99.

Nielsen, H. B. (2014). Systematic review of near-infrared spectroscopy determined cerebral oxygenation during non-cardiac surgery. Front. Physiol. 5:93. doi: 10. 3389/fphys.2014.00093

Nissilä, I., Kotilahti, K., Fallström, K., and Katila, T. (2002). Instrumentation for the accurate measurement of phase and amplitude in optical tomography. Rev. Sci. Instrum. 73, 3306-3312.

Nissilä, I., Noponen, T., Kotilahti, K., Fallström, K., Katila, T., Lipiäinen, L., et al. (2005). Instrumentation and calibration methods for the multichannel measurement of phase and amplitude in optical tomography. Rev. Sci. Instrum. 76:044302.

No, K.-S., Kwong, R., Chou, P. H., and Cerussi, A. (2008). Design and testing of a miniature broadband frequency domain photon migration instrument. J. Biomed. Opt. 13:050509. doi: 10.1117/1.2998473

Noponen, T., Kièiæ, D., Kotilahti, K., Kajava, T., Kähkönen, S., Nissilä, I., et al. (2005). Simultaneous diffuse near-infrared imaging of hemodynamic and oxygenation changes and electroencephalographic measurements of neuronal activity in the human brain. Proc. SPIE 5693, 179-190.

Noponen, T., Kotilahti, K., Toppila, J., Nissilä, I., Salmi, T., Kajava, T., et al. (2003). Near-infrared measurements of hemodynamic and oxygenation changes on the frontal cortex during breath holding, hyperventilation, and natural sleep. Proc. SPIE 4955, 124-133.

Ntziachristos, V., Brun, N., Feet, B., Greisen, G., and Chance, B. (1997). Oximetry with phase modulation NIR spectroscopy based on phase measurement only. Proc. SPIE 2979, 715-723.

Obrig, H. (2014). NIRS in clinical neurology: a 'promising' tool? Neuroimage 85, 535-546.

O'Sullivan, T. D., Cerussi, A. E., Cuccia, D. J., and Tromberg, B. J. (2012). Diffuse optical imaging using spatially and temporally modulated light. J. Biomed. Opt. 17:071311. doi: 10.1117/1.JBO.17.7.071311

Paisansathan, C., Hoffman, W. E., Gatto, R. G., Baughman, V. L., Mueller, M., and Charbel, F. T. (2007). Increased brain oxygenation during intubation-related stress. Eur. J. Anaesthesiol. 24, 1016-1020.

Parks, N. A., Maclin, E. L., Low, K. A., Beck, D. M., Fabiani, M., and Gratton, G. (2012). Examining cortical dynamics and connectivity with simultaneous single-pulse transcranial magnetic stimulation and fast optical imaging. Neuroimage 59, 2504-2510. doi: 10.1016/j.neuroimage.2011.08.097

Pham, T., Tgavalekos, K., Sassaroli, A., Blaney, G., and Fantini, S. (2019). Quantitative measurements of cerebral blood flow with near-infrared spectroscopy. Biomed. Opt. Express 10, 2117-2134. doi: 10.1364/BOE.10. 002117

Pham, T. H., Coquoz, O., Fishkin, J. B., Anderson, E., and Tromberg, B. J. (2000). Broad bandwidth frequency domain instrument for quantitative tissue optical spectroscopy. Rev. Sci. Instrum. 71, 2500-2513.

Pierro, M., Sassaroli, A., Bergethon, P. R., Ehrenberg, B. L., and Fantini, S. (2012). Phase-amplitude investigation of spontaneous low-frequency oscillations of cerebral hemodynamics with near-infrared spectroscopy: a sleep study in human subjects. Neuroimage 63, 1571-1584. doi: 10.1016/j.neuroimage.2012. 07.015

Power, S. D., Falk, T. H., and Chau, T. (2010). Classification of prefrontal activity due to mental arithmetic and music imagery using hidden Markov models and frequency domain near-infrared spectroscopy. J. Neural Eng. 7:026002. doi: $10.1088 / 1741-2560 / 7 / 2 / 026002$
Proulx, N., Samadani, A. A., and Chau, T. (2018a). Online classification of the nearinfrared spectroscopy fast optical signal for brain-computer interfaces. Biomed. Phys. Eng. Express 4:065010.

Proulx, N., Samadani, A. A., and Chau, T. (2018b). Quantifying fast optical signal and event-related potential relationships during a visual oddball task. Neuroimage 178, 119-128. doi: 10.1016/j.neuroimage.2018.05.031

Quaresima, V., and Ferrari, M. (2019). A mini-review on functional near-infrared spectroscopy (fNIRS): where do we stand, and where should we go? Photonics $6: 87$.

Radhakrishnan, H., Vanduffel, W., Deng, H. P., Ekstrom, L., Boas, D. A., and Franceschini, M. A. (2009). Fast optical signal not detected in awake behaving monkeys. Neuroimage 45, 410-419. doi: 10.1016/j.neuroimage.2008. 12.014

Rajan, K., Vijayakumar, V., Biswas, S. K., and Vasu, R. M. (2008). Implementation of a phase array diffuse optical tomography imager. Rev. Sci. Instrum. 79:084301. doi: 10.1063/1.2963042

Ramanujam, N., Du, C., Ma, H. Y., and Chance, B. (1998). Sources of phase noise in homodyne and heterodyne phase modulation devices used for tissue oximetry studies. Rec. Sci. Instrum. 69, 3042-3054.

Roblyer, D., O’Sullvan, T. D., Warren, R. V., and Tromberg, B. (2013). Feasibility of direct digital sampling for diffuse optical frequency domain spectroscopy in tissue. Meas. Sci. Technol. 24:045501.

Roche-Labarbe, N., Carp, S. A., Surova, A., Patel, M., Boas, D. A., Grant, P. E., et al. (2010). Noninvasive optical measures of $\mathrm{CBV}, \mathrm{StO}_{2}, \mathrm{CBF}$ index, and $\mathrm{rCMRO}_{2}$ in human premature neonates' brain in the first six weeks of life. Hum. Brain Mapp. 31, 341-352. doi: 10.1002/hbm.20868

Roche-Labarbe, N., Fenoglio, A., Aggarwal, A., Dehaes, M., Carp, S. A., Franceschini, M. A., et al. (2012). Near-infrared spectroscopy assessment of cerebral oxygen metabolism in the developing premature brain. J. Cereb. Blood Flow Metab. 32, 481-488. doi: 10.1038/jcbfm.2011.145

Roche-Labarbe, N., Fenoglio, A., Radhakrishnan, H., Kocienski-Filip, M., Carp, S. A., Dubb, J., et al. (2014). Somatosensory evoked changes in cerebral oxygen consumption measured non-invasively in premature neonates. Neuroimage 85 , 279-286. doi: 10.1016/j.neuroimage.2013.01.035

Ruesch, A., Schmitt, S., Yang, J., Smith, M. A., and Kainerstorfer, J. M. (2019). Fluctuations in intracranial pressure can be estimated non-invasively using near-infrared spectroscopy in non-human primates. J. Cereb. Blood Flow Metab. doi: 10.1177/0271678X19891359 [Epub ahead of print].

Sassaroli, A., Blaney, G., and Fantini, S. (2019). Dual-slope method for enhanced depth sensitivity in diffuse optical spectroscopy. J. Opt. Soc. Am. A 36, 17431761. doi: 10.1364/JOSAA.36.001743

Sassaroli, A., deFrederick, B., Tong, Y., Renshaw, P. F., and Fantini, S. (2006). Spatially weighted BOLD signal for comparison of functional magnetic resonance imaging and near-infrared imaging of the brain. Neuroimage 33, 505-514.

Sassaroli, A., and Fantini, S. (2004). Comment on the modified Beer-Lambert law for scattering media. Phys. Med. Biol. 49, N255-N257.

Sassaroli, A., Pifferi, A., Contini, D., Torricelli, A., Spinelli, L., Wabnitz, H., et al. (2014). Forward solvers for photon migration in the presence of highly and totally absorbing objects embedded inside diffusive media. J. Opt. Soc. Am. A 31, 460-469. doi: 10.1364/JOSAA.31.000460

Sassaroli, A., Tong, Y., Fabbri, F., Frederick, B., Renshaw, P., and Fantini, S. (2004). Functional mapping of the human brain with near-infrared spectroscopy in the frequency-domain. Proc. SPIE 5312, 371-377.

Sassaroli, A., Zheng, F., Hirshfield, L. H., Girouard, A., Solovey, E. T., Jacob, R. J. K., et al. (2008). Discrimination of mental workload levels in human subjects with functional near-infrared spectroscopy. J. Innov. Opt. Health Sci. 1, 227-237. doi: 10.3389/fnhum.2013.00935

Sawosz, P., and Liebert, A. (2019). Method to improve the depth sensitivity of diffuse reflectance measurements to absorption changes in optically turbid medium. Biomed. Opt. Express 10, 5031-5041. doi: 10.1364/BOE.10.00 5031

Schmitt, J. M., Knûttel, A., and Knutson, J. R. (1992). Interference of diffusive light waves. J. Opt. Soc. Am. A 9, 1832-1843.

Scholkmann, F., Gerber, U., Wolf, M., and Wolf, U. (2013a). End-tidal CO2: an important parameter for a correct interpretation in functional brain studies using speech tasks. Neuroimage 66, 71-79. doi: 10.1016/j.neuroimage.2012. 10.025 
Scholkmann, F., Wolf, M., and Wolf, U. (2013b). The effect of inner speech on arterial $\mathrm{CO}_{2}$, cerebral hemodynamics and oxygenation - A functional NIRS study. Adv. Exp. Med. Biol. 789, 81-87. doi: 10.1007/978-1-4614-7411-1_12

Scholkmann, F., Hafner, T., Metz, A. J., Wolf, M., and Wolf, U. (2017). Effect of short-term colored-light exposure on cerebral hemodynamics and oxygenation, and systemic physiological activity. Neurophotonics 4:045005. doi: 10.1117/1. NPh.4.4.045005

Scholkmann, F., Klein, S., Gerber, U., Wolf, M., and Wolf, U. (2014a). Cerebral hemodynamic and oxygenation changes induced by inner and heard speech: a study combining functional near-infrared spectroscopy and capnography. J. Biomed. Opt. 19:017002. doi: 10.1117/1.JBO.19.1.017002

Scholkmann, F., Kleiser, S., Jaakko Metz, A., Zimmermann, R., Mata Pavia, J., Wolf, U., et al. (2014b). A review on continuous wave functional near-infrared spectroscopy and imaging instrumentation and methodology. Neuroimage 85 , $6-27$.

Schwarz, C. E., Preusche, A., Wolf, M., Poets, C. F., and Franz, A. R. (2018). Prospective observational study on assessing the hemodynamic relevance of patent ductus arteriosus with frequency domain near-infrared spectroscopy. BMC Pediatr. 18:66. doi: 10.1186/s12887-018-1054-6

Sevick, E. M., Chance, B., Leigh, J., Nioka, S., and Maris, M. (1991). Quantitation of time- and frequency-resolved optical spectra for the determination of tissue oxygenation. Anal. Biochem. 195, 330-351.

Smith, M. (2011). Shedding light on the adult brain: a review of the clinical applications of near-infrared spectroscopy. Philos. Trans. R. Soc. A 369, 44524469. doi: 10.1098/rsta.2011.0242

Solovey, E. T., Lalooses, F., Chauncey, K., Weaver, D., Parasi, M., Scheutz, M., et al. (2011). "Sensing cognitive multitasking for a brain-based adaptive user interface," in Proceedings of the ACM Conference on Human Factors in Computing Systems CHI'11, (New York, NY: ACM Press), 383-392.

Srinivasan, S., Davis, S. C., and Carpenter, C. M. (2011). "Diffuse optical tomography using CW and frequency domain imaging systems," in Handbook of Biomedical Optics, eds D. A. Boas, C. Pitris, and N. Ramanujam (Boca Raton, FL: CRC Press), 373-394.

Steinbrink, J., Kempf, F. C. D., Villringer, A., and Obrig, H. (2005). The fast optical signal - Robust or elusive when non-invasively measured in the human adult? Neuroimage 26, 996-1008.

Steinbrink, J., Kohl, M., Obrig, H., Curio, G., Syré, F., Thomas, F., et al. (2000). Somatosensory evoked fast optical intensity changes detected non-invasively in the adult human head. Neurosci. Lett. 291, 105-108.

Sthalekar, C. C., and Joyner Koomson, V. (2013). A CMOS sensor for measurement of cerebral optical coefficients using non-invasive frequency domain near infrared spectroscopy. IEEE Sens. J. 13, 3166-3174.

Sultan, E., Najafizadeh, L., Gandjbakhche, A. H., Pourrezaei, K., and Daryoush, A. (2013). Accurate optical parameter extraction procedure for broadband nearinfrared spectroscopy of brain matter. J. Biomed. Opt. 18:017008. doi: 10.1117/ 1.JBO.18.1.017008

Sun, B., Zhang, L., Gong, H., Sun, J., and Luo, Q. (2014). Detection of optical neuronal signals in the visual cortex using continuous wave near-infrared spectroscopy. Neuroimage 87, 190-198. doi: 10.1016/j.neuroimage.2013.11.003

Suzuki, S., Takasaki, S., Ozaki, T., and Kobayashi, Y. (1999). Tissue oxygenation monitor using NIR spatially resolved spectroscopy. Proc. SPIE 3597, 582-592.

Tgavalekos, K. T., Kainerstorfer, J. M., Sassaroli, A., and Fantini, S. (2016). Bloodpressure-induced oscillations of deoxy- and oxyhemoglobin concentrations are in-phase in the healthy breast and out-of-phase in the healthy brain. J. Biomed. Opt. 21:101410. doi: 10.1117/1.JBO.21.10.101410

Tian, F., Jenks, C., Potter, D., Miles, D., and Raman, L. (2017). Regional cerebral abnormalities measured by frequency-domain near-infrared spectroscopy in pediatric patients during extracorporeal membrane oxygenation. ASAIO J. 63, e52-e59. doi: 10.1097/MAT.0000000000000453

Tong, Y., Rooney, E. J., Bergethon, P. R., Martin, J. M., Sassaroli, A., Ehrenberg, B. L., et al. (2005). Studying brain function with near-infrared spectroscopy concurrently with electroencephalography. Proc. SPIE 5693, 444-449.

Torjesen, A., Istfan, R., and Roblyer, D. (2017). Ultrafast wavelength multiplexed broad bandwidth digital diffuse optical spectroscopy for in vivo extraction of tissue optical properties. J. Biomed. Opt. 22:036009. doi: 10.1117/1.JBO.22.3. 036009

Toronov, V., D'Amico, E., Hueber, D., Gratton, E., Barbieri, B., and Webb, A. (2004). Optimization of the phase and modulation depth signal-to-noise ratio for near-infrared spectroscopy of the biological tissue. Proc. SPIE 5474, 281-284.

Toronov, V., Webb, A., Choi, J. H., Wolf, M., Safonova, L., Wolf, U., et al. (2001). Study of local cerebral hemodynamics by frequency-domain near-infrared spectroscopy and correlation with simultaneously acquired functional magnetic resonance imaging. Opt. Express 9, 417-427.

Torricelli, A., Contini, D., Pifferi, A., Caffini, M., Re, R., Zucchelli, L., et al. (2014). Time domain functional NIRS imaging for human brain mapping. Neuroimage 85, 28-50. doi: 10.1016/j.neuroimage.2013.05.106

Tromberg, B. J., Svaasand, L. O., Tsay, T. T., and Haskell, R. C. (1993). Properties of photon density waves in multiple-scattering media. Appl. Opt. 32, 607-616. doi: 10.1364/AO.32.000607

Tse, C.-Y., and Penney, T. B. (2007). Preattentive change detection using the event-related optical signal. IEEE Eng. Med. Biol. Mag. 26, 52-58.

Tse, C.-Y., Rinne, T., Ng, K. K., and Penney, T. B. (2013). The functional role of the frontal cortex in pre-attentive auditory change detection. Neuroimage 83 , 870-879. doi: 10.1016/j.neuroimage.2013.07.037

Tse, C.-Y., Tien, K. R., and Penney, T. B. (2006). Event-related optical imaging reveals the temporal dynamics of right temporal and frontal cortex activation in pre-attentive change detection. Neuroimage 29, 314-320.

Tuchin, V. (2007). Tissue Optics: Light Scattering Methods, and Instruments for Medical Diagnosis, 2nd Edn. Bellingham, WA: SPIE Press, 463-482.

Virtanen, J., Noponen, T., and Meriläinen, P. (2009). Comparison of principal and independent component analysis in removing extracerebral interference from near-infrared spectroscopy signals. J. Biomed. Opt. 14:054032. doi: 10.1117/1. 3253323

Wang, L. V., and Wu, H. I. (2007). Biomedical Optics: Principles and Imaging. Hoboken, NJ: Wiley, 253-275.

Watzman, H. M., Kurth, C. D., Montenegro, L. M., Rome, J., Steven, J. M., and Nicolson, S. C. (2000). Arterial and venous contributions to near-infrared cerebral oximetry. Anesthesiology 93, 947-953.

Wilson, B. C., Sevick, E. M., Patterson, M. S., and Chance, B. (1992). Timedependent optical spectroscopy and imaging for biomedical applications. Proc. IEEE 80, 918-930.

Wolf, M., Naulaers, G., van Bel, F., Kleiser, S., and Greisen, G. (2012). A review of near infrared spectroscopy for term and preterm newborns. J. Near Infrared Spectrosc. 20, 43-55.

Wolf, M., Wolf, U., Choi, J. H., Gupta, R., Safonova, L. P., Paunescu, L. A., et al. (2002a). Functional frequency-domain near-infrared spectroscopy detects fast neuronal signal in the motor cortex. Neuroimage 17, 1868-1875.

Wolf, M., Wolf, U., Choi, J. H., Toronov, V., Paunescu, L. A., Michalos, A., et al. (2003). Fast cerebral functional signal in the 100-ms range detected in the visual cortex by frequency-domain near-infrared spectrophotometry. Psychophysiology 40, 521-528.

Wolf, M., Wolf, U., Toronov, V., Michalos, A., Paunescu, L. A., Choi, J. H., et al. (2002b). Different time evolution of oxyhemoglobin and deoxyhemoglobin concentration changes in the visual and motor cortices during functional stimulation: a near-infrared spectroscopy study. Neuroimage 16, 704-712.

Yang, M., Yang, Z., Yuan, T., Feng, W., and Wang, P. (2019). A systemic review of functional near-infrared spectroscopy for stroke: current application and future directions. Front. Neurol. 10:58. doi: 10.3389/fneur.2019. 00058

Yang, R., and Dunn, J. F. (2015). Reduced cortical microvascular oxygenation in multiple sclerosis: a blinded, case-controlled study using a novel quantitative near-infrared spectroscopy method. Sci. Rep. 5:16477. doi: 10.1038/srep16477

Yang, Y., Liu, H., Li, X., and Chance, B. (1997). Low-cost frequency-domain photon migration instrument for tissue spectroscopy, oximetry, and imaging. Opt. Eng. 36, 1562-1569.

Yu, G., Durduran, T., Furuya, D., Greenberg, J. H., and Yodh, A. G. (2003). Frequency-domain multiplexing system for in vivo diffuse light measurements of rapid cerebral hemodynamics. Appl. Opt. 42, 2931-2939.

Yun, R., and Joyner Koomson, V. (2013). A novel CMOS frequency-mixing transimpedance amplifier for frequency domain near infrared spectroscopy. IEEE Trans. Circuits Syst. 60, 84-94.

Zhang, G., Katz, A., Alfano, R. R., Kofinas, A. D., Kofinas, D. A., Stubblefield, P. G., et al. (2000). Brain perfusion monitoring with frequency-domain and continuous-wave near-infrared spectroscopy: a cross-correlation study in newborn piglets. Phys. Med. Biol. 45, 3143-3158. 
Zhang, Z., Bolz, N., Laures, M., Oremek, M., Schmidt, C., Qi, M., et al. (2017). Cerebral blood volume and oxygen supply uniformly increase following various intrathoracic pressure strain. Sci. Rep. 7:8344.

Zhang, Z., Sun, B., Gong, H., Zhang, L., Sun, J., Wang, B., et al. (2012). A fast neuronal signal-sensitive continuous-wave near-infrared imaging system. Rev. Sci. Instrum. 83:094301.

Zhao, J., Ding, H. S., Hou, X. L., Zhou, C. L., and Chance, B. (2005). In vivo determination of the optical properties of infant brain using frequency-domain near-infrared spectroscopy. J. Biomed. Opt. 10:024028.

Zhao, Y., Applegate, M. B., Istfan, R., Pande, A., and Roblyer, D. (2018). Quantitative real-time pulse oximetry with ultrafast frequency-domain diffuse optics and deep neural network processing. Biomed. Opt. Express 9, 5997-6008. doi: 10.1364/BOE.9.005997
Zimmermann, B. B., Fang, Q., Boas, D. A., and Carp, S. A. (2016). Frequency domain near-infrared multiwavelength imager design using high-speed, direct analog-to-digital conversion. J. Biomed. Opt. 21:016010.

Conflict of Interest: The authors declare that the research was conducted in the absence of any commercial or financial relationships that could be construed as a potential conflict of interest.

Copyright (C) 2020 Fantini and Sassaroli. This is an open-access article distributed under the terms of the Creative Commons Attribution License (CC BY). The use, distribution or reproduction in other forums is permitted, provided the original author(s) and the copyright owner(s) are credited and that the original publication in this journal is cited, in accordance with accepted academic practice. No use, distribution or reproduction is permitted which does not comply with these terms. 